CANTUA. 2016; 15:12- 46

Fecha de recepción: 24.09.2016

Fecha de aceptación: 19.12.2016

\title{
Diversidad de aves de los bosques montanos en el departamento del Cusco
}

Birds diversity of the montane rainforest in the department of Cusco

\author{
Norma Jara-Moscoso ${ }^{1, a}$, Olintho Aguilar-Condemayta ${ }^{1}$, Luis Ayma-Cornejo ${ }^{1}$, Karina \\ Vargas-Serrano $^{1}$, Karol Mejía-Espinoza ${ }^{1}$, Daniela S. Olivera-Aguirre ${ }^{1} \&$ José Antonio \\ Ochoa $^{1,2}$
}

1 Escuela Profesional de Biología, Facultad de Ciencias, Universidad Nacional de San Antonio Abad del Cusco., Cusco, Perú.

${ }^{2}$ Museo de Biodiversidad del Perú. Cusco, Perú.

${ }^{\mathrm{a}}$ ORCID. 0000-0001-5302-41-43

\section{RESUMEN}

Se presenta un estudio de la diversidad de aves de bosques montanos en el departamento del Cusco basado en revisión de colecciones de Museo y bibliografía. 671 especies de aves fueron registradas en total para los bosques montanos de Cusco ubicados entre los 700 a $3800 \mathrm{~m}$ de altitud: 584 especies para el Parque Nacional del Manu, 325 para Machupicchu, 150 para Choquequirao, 131 para Yanatile y 190 para el valle de Marcapata. El grado de similitud de Jaccard para todos los casos fue menor al 50\%. A pesar que existe varias investigaciones en el Manu y Machupicchu, existen vacíos de información en la diversidad de aves en los valles de Marcapata, Yanatile, Umasbamba, la cuenca media del río Urubamba, y Choquequirao.

PALABRAS CLAVE: Biodiversidad, aves, Andes, Amazonía, museo.

\section{ABSTRACT}

A study of the diversity of the montane rainforest birds in the department of Cusco based on 
review of Museum collections and bibliography is presented. 671 bird species were recorded in total for the montane forests of Cusco located between 700 to $3800 \mathrm{~m}$ asl: 584 species from the Manu National Park, 325 from Machupicchu, 150 from Choquequirao, 131 from Yanatile and 190 from the Marcapata Valley. The Jaccard similarity scores, for all cases, were less than $50 \%$. Although, there are several researches in Manu and Machupicchu, there are gaps of information on the diversity of birds in others valleys from Cusco as Marcapata, Yanatile, Umasbamba, the middle basin of the Urubamba River, and Choquequirao.

KEY WORDS: Biodiversity, birds, Andes, Amazonia, museum.

\section{INTRODUCCIÓN}

El Perú ocupa el segundo lugar en diversidad de aves a nivel mundial con más de 1850 especies registradas en todo el país, la cual se corresponde con la enorme variabilidad ambiental que ocurre en el Perú en sus diferentes regiones naturales, tanto en la costa, Andes y Amazonía. Uno de los lugares más importantes en términos de diversidad ornitológica corresponde a los bosques montanos que albergan gran cantidad de especies endémicas (Plenge, 2016).

Los bosques montanos orientales al sur de Perú se ubican aproximadamente sobre los 1500 metros y limitan en su parte superior con formaciones de puna o pajonal alrededor de los $3800 \mathrm{~m}$ de altitud. Originalmente existió una franja boscosa continua casi de 1500 kilómetros de largo desde los $5^{\circ}$ hasta los $15^{\circ}$ y actualmente se encuentra fragmentada, en parte por carretera y agricultura, ej. valle de Umasbamba, Kosñipata, Marcapata, entre otros (Young, 1992, 1993). Aunque la literatura sobre biodiversidad de bosques montanos del Cusco sobre las aves no es escasa, ésta se encuentra dispersa y los trabajos corresponden principalmente a estudios de algunos taxa, trabajos de inventarios faunísticos dentro de áreas naturales protegidas y la zona del Bajo Urubamba, y trabajos biogeográficos y de aspectos ecológicos (Terborgh, 1971, 1977; Fjieldsa, 1992; O`Neil, 1992). Esta información se encuentra compilada en el libro de Aves de Perú y el libro de los Altos Andes (Fjeldså \& Krabbe, 1990; Schulenberg et al., 2010). Dentro del ámbito del departamento del Cusco destacan las expediciones de la Universidad de Yale realizadas entre 1911 y 1915, cuyos resultados para el caso de las aves fueron publicados en 1921 por Chapman (1921). Trabajos más recientes en áreas aledañas o que involucran alguna localidad cercana: Ceballos Bendezú, 1994; Walker \& Ricalde, 1998; Mujica, 1998; Ochoa Mendieta, 1987, 1997; Walker et al, 2006; Walker, 2015; Walker \& Stolz, 2017, Atauchi, 2015; GORE-Cusco, 2010.

El presente trabajo presenta un panorama de la biodiversidad de los bosques montanos del departamento de Cusco, teniendo como modelo al grupo de las aves, al ser el grupo que 
probablemente tiene mayor información con respecto a otros taxa de vertebrados e invertebrados. El objetivo final fue compilar la información de la riqueza de especies en base a datos bibliográficos y revisión de colecciones de museos, así como conocer el grado de diferenciación que pueda existir en la diversidad ornitológica de los bosques montanos del departamento del Cusco.

\section{METODOLOGÍA}

\section{Recopilación bibliográfica}

Se realizaron búsquedas exhaustivas de documentación y material bibliográfico por internet en bases de datos de libre acceso (Free E-journals, Freely Accessible Journals) o mediante subscripciones a Bio One, JSTOR, Blackwell-Synergy, Directory of Open Access Journals, y Elsevier ScienceDirect. Para las búsquedas por Internet usamos palabras clave como aves, bosques montanos, bosque nublado, Kosñipata, Acjanaco, Abra de Málaga, Manu, Machupicchu, entre otros y seleccionamos únicamente los documentos con información sobre aves de bosques montanos y aquellas que tengan referencias citadas en la bibliografía que incluyan información de relevancia sobre especies de aves. También se visitó la base de datos de EBird (2018) y de la IUCN (2016). Asimismo se revisó las bibliotecas locales, especialmente de la UNSAAC, para obtener datos de tesis y seminarios.

\section{Creación de una base de datos}

En base a la revisión bibliográfica se ha confeccionado una base de datos sobre la ocurrencia de las especies de aves. Para la sistematización de la información se tuvo en cuenta la identidad específica de la especie, localidad, altitud, procedencia de la información y fecha, y presencia o ausencia en los diferentes bosques montanos. Se discriminó la información en los siguientes bosques que corresponden a los principales valles interandinos que van hacía el lado oriental de los Andes:

- Marcapata - Quincemil

- $\quad$ Acjanaco - Kosñipata

- Amparaes - Yanatile

- Machupicchu

- Choquequirao 


\section{Revisión de colecciones biológicas}

Para complementar la información proveniente de la literatura, realizamos una revisión de las colecciones de fauna del Museo de Historia Natural de la UNSAAC, Gabinete de Zoología de la Facultad de Ciencias (Ochoa Mendieta, 1997) y del Museo de la Universidad Nacional San Agustín de Arequipa (MUSA, 2017).

\section{Análisis de datos}

El análisis estuvo enfocado a la obtención de datos de composición y riqueza de especies; los cálculos de índices cualitativos basados en riqueza de especies se realizaron con el programa PAST (Paleontological Statistics) versión 1.90; así como Microsoft Office Excel, para confeccionar los gráficos respectivos. Para conocer el grado de similitud (diversidad beta) de las diferentes zonas de estudio se utilizó el coeficiente de similitud de Jaccard. Este índice relaciona el número de especies compartidas entre dos localidades con el número total de especies exclusivas (Moreno, 2001) con la ecuación:

$\mathrm{I}_{\mathrm{j}}=\mathrm{c} /(\mathrm{a}+\mathrm{b}-\mathrm{c})$

Donde: $\mathrm{a}=$ número de especies en la localidad $\mathrm{A} ; \mathrm{b}=$ número de especies en la localidad $\mathrm{B} ; \mathrm{c}=$ número de especies presentes en ambas localidades.

\section{RESULTADOS}

Se registraron un total de 671 especies de aves para bosques montanos de la región de Cusco que incluye ecosistemas desde los $700 \mathrm{~m}$ de altitud en el límite con la Amazonía hasta los 3800 m en el límite con la Puna (Anexo 1). Las familias Tyrannidae y Thraupidae resultaron las más diversas con 112 y 86 especies respetivamente, destacando también las familias Trochilidae con 62 especies y Furnariidae con 56 especies como las más representativas. El número total de familias registradas fue de 56. Esta diversidad representa el $36 \%$ de total de aves presentes en el Perú.

El bosque enano ubicado entre los 2500 - $3800 \mathrm{~m}$, alberga 251 especies pertenecientes a 44 familias de aves; del mismo modo el bosque nublado que se ubica entre los $1500-2500 \mathrm{~m}$ aproximadamente presenta 355 especies en 48 familias; mientras que el bosque lluvioso, ubicado entre los 700 - $1500 \mathrm{~m}$ de altitud, presenta 356 especies con 52 familias (tabla 1). 
Tabla 1.

Número de especies y familias de aves por tipo de bosque y sectores de bosque montano en el departamento del Cusco.

\begin{tabular}{|c|c|c|c|c|c|c|c|c|}
\hline \multirow[b]{2}{*}{ Número } & \multicolumn{3}{|c|}{ Tipo de bosque } & \multicolumn{5}{|c|}{ Sector /valle } \\
\hline & $\begin{array}{c}\text { Bosque } \\
\text { Enano } \\
(3800- \\
2500)\end{array}$ & $\begin{array}{c}\text { Bosque } \\
\text { Nublado } \\
(2500- \\
1500)\end{array}$ & $\begin{array}{c}\text { Bosque } \\
\text { Lluvioso } \\
(1500- \\
700)\end{array}$ & Manu & Machupicchu & Choquequirao & Yanatile & Marcapata \\
\hline Especies & 251 & 355 & 356 & 584 & 325 & 150 & 131 & 190 \\
\hline Familias & 44 & 48 & 52 & 54 & 48 & 37 & 42 & 45 \\
\hline
\end{tabular}

El Parque Nacional del Manu, cuyo sector de bosque montano corresponde al Valle de Kosñipata desde la zona de Tres Cruces hasta Pillcopata, presenta 584 especies, siendo el área de bosque montano con mayor diversidad en la región de Cusco, esto se debe a que existe una continuidad boscosa sin mayor alteración por actividades humanas, donde los tres sectores de los bosques montanos están presentes a lo largo de la gradiente altitudinal.

En los bosques montanos del río Urubamba que corresponde principalmente al Santuario Histórico de Machupicchu se han registrado 325 especies, lo cual correspondería al segundo sector con mayor diversidad de aves en bosques montanos en el Cusco, no obstante el SH Machupicchu no cuenta con sectores de bosque lluvioso. Otros valles donde se tienen registros de aves presentan menor diversidad: Valle de Marcapata con 190 especies, Yanatile con 131 especies y 150 especies en Choquequirao.

El número de familias registradas también difiere en los distintos sectores, así en la zona del PN Manu se han registrado 54 familias de aves, en Machupicchu 48, mientras que los otros valles hay varias familias que no han sido registradas hasta la fecha, contando solamente con 37, 42 y 45 familias en Choquequirao, Yanatile y Marcapata (tabla 2). 
Tabla 2.

Diversidad de aves a nivel de especies por familias para los bosques montanos del departamento del Cusco.

\begin{tabular}{|c|c|c|c|c|c|}
\hline & Familia & $\begin{array}{c}\mathrm{N}^{\circ} \mathrm{de} \\
\text { especies } \\
\text { total }\end{array}$ & $\begin{array}{c}\text { Bosque } \\
\text { Enano } \\
(3800-2500)\end{array}$ & $\begin{array}{c}\text { Mosque } \\
\text { Montano } \\
(2500-1500)\end{array}$ & $\begin{array}{c}\text { Bosque } \\
\text { Lluvioso } \\
(1500-700)\end{array}$ \\
\hline 1 & Tinamidae & 10 & 4 & 4 & 5 \\
\hline 2 & Anatidae & 3 & 2 & 1 & 1 \\
\hline 3 & Cracidae & 5 & 1 & 3 & 2 \\
\hline 4 & Odontophoridae & 2 & 1 & 1 & 1 \\
\hline 5 & Phalacrocoracidae & 1 & & 1 & 1 \\
\hline 6 & Ardeidae & 2 & 1 & 1 & 1 \\
\hline 7 & Thinocoridae & 1 & 1 & & \\
\hline 8 & Cathartidae & 3 & 1 & 1 & 2 \\
\hline 9 & Accipitridae & 23 & 5 & 11 & 15 \\
\hline 10 & Eurypygidae & 1 & & 1 & 1 \\
\hline 11 & Falconidae & 10 & 6 & 6 & 5 \\
\hline 12 & Rallidae & 1 & & & 1 \\
\hline 13 & Scolopacidae & 7 & 6 & 5 & 5 \\
\hline 14 & Laridae & 1 & 1 & & \\
\hline 15 & Columbidae & 13 & 5 & 6 & 6 \\
\hline 16 & Cuculidae & 5 & 1 & 5 & 3 \\
\hline 17 & Tytonidae & 1 & 1 & 1 & 1 \\
\hline 18 & Strigidae & 13 & 5 & 10 & 6 \\
\hline 19 & Steatornithidae & 1 & & 1 & \\
\hline 20 & Nictybiidae & 1 & & 1 & 1 \\
\hline 21 & Caprimulgidae & 7 & 2 & 6 & 4 \\
\hline 22 & Apodidae & 8 & 3 & 5 & 4 \\
\hline 23 & Trochilidae & 62 & 26 & 34 & 34 \\
\hline 24 & Trogonidae & 4 & 1 & 4 & 1 \\
\hline 25 & Alcedinidae & 2 & & & 2 \\
\hline 26 & Momotidae & 2 & 1 & 2 & 1 \\
\hline 27 & Bucconidae & 7 & 1 & 4 & 6 \\
\hline 28 & Galbulidae & 1 & & & 1 \\
\hline 29 & Capitonidae & 4 & 1 & 2 & 2 \\
\hline 30 & Ramphastidae & 6 & 1 & 5 & 3 \\
\hline 31 & Picidae & 17 & 3 & 5 & 10 \\
\hline 32 & Psittacidae & 15 & 7 & 1 & 8 \\
\hline 33 & Thamnophilidae & 38 & & 7 & 34 \\
\hline 34 & Conopophagidae & 2 & & 1 & 2 \\
\hline 35 & Grallariidae & 8 & 4 & 3 & 2 \\
\hline 36 & Rhinocryptidae & 6 & 5 & 2 & 1 \\
\hline 37 & Formicariidae & 3 & 2 & & 1 \\
\hline 38 & Furnariidae & 56 & 24 & 22 & 34 \\
\hline 39 & Pipridae & 7 & & 3 & 7 \\
\hline
\end{tabular}




\begin{tabular}{|l|l|c|c|c|c|}
40 & Tyrannidae & 112 & 37 & 62 & 70 \\
\hline 41 & Cotingidae & 12 & 4 & 5 & 7 \\
\hline 42 & Tityridae & 8 & 1 & 2 & 7 \\
\hline 43 & Vireonidae & 5 & & 3 & 3 \\
\hline 44 & Corvidae & 3 & 1 & 2 & 2 \\
\hline 45 & Hirundinidae & 9 & 6 & 4 & 5 \\
\hline 46 & Troglodytidae & 10 & 6 & 6 & 4 \\
\hline 47 & Polioptilidae & 1 & & & 1 \\
\hline 48 & Cinclidae & 1 & 1 & 1 & 1 \\
\hline 49 & Turdidae & 12 & 6 & 9 & 3 \\
\hline 50 & Motacillidae & 1 & 1 & & \\
\hline 51 & Thraupidae & 86 & 45 & 57 & 22 \\
\hline 52 & Emberizidae & 15 & 8 & 10 & 5 \\
\hline 53 & Cardinalidae & 9 & 3 & 6 & 4 \\
\hline 54 & Parulidae & 15 & 5 & 13 & 4 \\
\hline 55 & Icteridae & 7 & 2 & 4 & 3 \\
\hline 56 & Fringillidae & 6 & 3 & 6 & 1 \\
\hline Total & & 671 & 251 & 355 & 356 \\
\hline
\end{tabular}

El grado de remplazamiento de la riqueza de especies (diversidad beta), en términos de similitud/disimilitud es muy evidente a nivel altitudinal, prácticamente existe un reemplazo total de especies entre el bosque enano ubicado por encima de los $2500 \mathrm{~m}$ de altitud, con respecto al bosque lluvioso ubicado por debajo de los $1500 \mathrm{~m}$. El grado de similitud entre el bosque enano y bosque de nubes es apenas de $34 \%$, mientras que entre bosque nuboso y bosque lluvioso es del $24 \%$ (tabla 3 ).

Tabla 3.

Grado de Similitud (índice de Jaccard) a nivel de riqueza de especies de aves por tipo de bosque

\begin{tabular}{c|ccc}
\hline \multicolumn{1}{c|}{ Tipo de bosque } & $\begin{array}{c}\text { Bosque } \\
\text { Enano }\end{array}$ & $\begin{array}{c}\text { Bosque de } \\
\text { Nubes }\end{array}$ & $\begin{array}{c}\text { Bosque } \\
\text { lluvioso }\end{array}$ \\
\hline Bosque Enano $(2500-3800 \mathrm{~m})$ & 1 & 0.34 & 0.05 \\
$\begin{array}{l}\text { Bosque de Nubes }(1500-2500 \\
\mathrm{m})\end{array}$ & 0.34 & 1 & 0.24 \\
Bosque lluvioso $(700-1500 \mathrm{~m})$ & 0.05 & 0.24 & 1 \\
\hline
\end{tabular}

Esto significa que cada uno de los sectores a nivel altitudinal presenta un alto grado de endemismo, siendo que el bosque enano tiene mayor influencia de elementos andinos, y a medida que se desciende en altitud la fauna recibe mayor influencia de la Amazonía.

El grado de disimilitud entre los diferentes valles es también marcado, en todos los casos mayor a 50\%. Los sectores con mayor similitud corresponde al PN Manu comparado con el SH 
Machupicchu con $41 \%$ de similitud (tabla 4). Asimismo Machupicchu y Choquequirao tienen una similitud de 35\%. La comparación entre los otros valles presentan valores inferiores al 30\% (tabla 4).

Tabla 4.

Grado de Similitud (índice de Jaccard) a nivel de riqueza de especies de aves por sector y/o valle evaluado.

\begin{tabular}{l|ccccc}
\hline & Manu & Machupicchu & Choquequirao & Yanatile & Marcapata \\
\hline Manu & 1 & 0.41 & 0.2 & 0.19 & 0.26 \\
Machupicchu & 0.41 & 1 & 0.35 & 0.27 & 0.23 \\
Choquequirao & 0.2 & 0.35 & 1 & 0.21 & 0.2 \\
Yanatile & 0.19 & 0.27 & 0.21 & 1 & 0.26 \\
Marcapata & 0.26 & 0.23 & 0.2 & 0.26 & 1 \\
\hline
\end{tabular}

Esta gran diferencia en la riqueza de especies entre Kosñipata, Machupicchu y los otros valles de selva alta, así como su grado de similitud/disimilitud se debe a dos factores: i) vacíos de información y ii) destrucción de los ecosistemas. El número de investigaciones es menor en estos valles de selva alta de la región de Cusco en comparación con el PN Manu (valle de Kosñipata) y el SH Machupicchu, lo cual indica un gran vacío de información en estos sectores y que podrían existir más registros (mayor riqueza) si se incrementa más evaluaciones y/o estudios de aves. También evidente que durante cientos de años, algunos sectores de bosques montanos de Cusco han sufrido una alto grado de deforestación, como por ejemplo en el valle del río Urubamba donde existe una discontinuidad boscosa desde Santa Teresa hasta el encuentro con el río Yanatile, existiendo sólo algunos parches de bosques aislados.

\section{CONCLUSIONES}

En conclusión podemos decir que la diversidad de aves de los bosques montanos de Cusco es muy alta, representando más de un tercio de total de aves del Perú, siendo el valle de Kosñipata el que presenta mayor riqueza de especies. Un segundo lugar con mayor número de investigaciones es el SH Machupicchu, donde se han registrado 325 especies. Asimismo, existe una similaridad en la composición de especies de aves en todos los sectores evaluados de bosques montanos menor al 50\%, no obstante, con esos valores resulta ser más semejante la ornitofauna de Marcapata con Manu y Machupicchu, siendo que Machupicchu también presente cierta semejanza con Choquequirao. A pesar de ello debemos indicar que existe un gran vació de información en la mayoría de valles tropicales de la región como la zona de Valles de Umasbamba, Yanatile, Marcapata, Choquequirao y la cuenca media del río Urubamba. 
Finalmente es evidente que la deforestación histórica en ciertos valles de selva alta, ha ocasionado una gran pérdida de biodiversidad, especialmente en el sector de bosque lluvioso entre los 700 y $1500 \mathrm{~m}$, justamente donde se encuentran los principales valles tropicales que se han dedicado históricamente a fines agrícolas (Valle del Mapacho, Lacco, La Convención, Vilcabamba, etc). La principal consecuencia de la deforestación es la falta de conectividad de los ecosistemas, resultando que muchos sectores actualmente se encuentren aislados, como es el caso del SH Machupicchu.

\section{Autor corresponsal:}

Norma Jara- Moscoso

Correo electrónico: mary.jara@unsaac.edu.pe

\section{Referencia bibliográfica}

Atauchi-Rojas, P.J. (2015). Variación Espacial de las Especies de Aves en el Valle de Yanatile, Provincia de Calca, Departamento de Cusco. Seminario de investigación. Facultad de Ciencias, Escuela Profesional de Biología, Universidad Nacional de San Antonio Abad del Cusco, 55 pp.

Ceballos-Bendezú, I. (1994). Fauna del Santuario Histórico de Machupicchu en Machupicchu, En: R. Chevarría (Ed.) Devenir Histórico y Cultural. 79-89 pp.

Chapman, F.M. (1921). The distribution of bird life in the Urubamba Valley of Peru. A report on the birds collected by the Yale University-National Geographic Society's Expeditions. Bulletin of the United States National Museum, 1-138.

Ebird. ( 2016). Base de datos. http://ebird.org/ebird/hotspot/L782919.

Fjeldså, J. (1992). Un análisis biogeográfico de la avifauna de los bosques de queuña (Polylepis) de los Andes y su relevancia para establecer prioridades de conservación. En: Biogeografía, Ecología y Conservación del Bosque Montano en el Perú. Museo de Historia Natural UNMSM. 21. Lima. Perú.

Fjeldså, J. \& Krabbe, N. (1990). Birds of the high Andes. Zoological Museum, University of Copenhagen and Apollo Books, Copenhagen.

Gobierno Regional Cusco. (2010). Guía de Campo del Área Prioritaria de Conservación Regional Corredor Biológico Marcapata - Camanti. Cusco. Perú.

IUCN. (2016). IUCN Red List of Threatened Species Version 2016.1. www.iucnredlist.org..

Moreno, C.E. (2001). Métodos para medir la biodiversidad. M\&T-Manuales y Tesis SEA, vol.1. Zaragoza, $84 \mathrm{pp}$.

Mujica, O.D. (1998). Evaluación Preliminar de la Ornitofauna de Pillahuata. Seminario Curricular, Facultad de Ciencias Biológicas, Universidad Nacional de San Antonio Abad del 
Cusco.

Museo de Historia Natural de la Universidad Nacional de San Agustín. (2017). Colección de pieles y huesos de aves del Cusco. Arequipa, MUSA.

Ochoa-Mendieta, O. (1987). Contribución al conocimiento de la Ornitología Regional (tesis doctoral). Facultad de Ciencias Biológicas y Geografía, Universidad Nacional de San Antonio Abad del Cusco, Perú.

Ochoa-Mendieta, O. (1997). Catálogo de vertebrados del gabinete de Zoología de la UNSAAC. Facultad de Ciencias Biológicas, Universidad Nacional de San Antonio Abad del Cusco.

O’Neill, J.P. (1992). A general overview of the montane avifauna of Perú. En: Biogeografía, Ecología y Conservación del Bosque Montano en el Perú. Museo de Historia Natural. UNMSM. 21. Lima. Perú. Pp. 47-55.

Plenge, M.A. (2017). List of the birds of Peru. Unión de Ornitólogos del Perú: https://sites.google.com/site/boletinunop/checklist. Acceso 04/12/2017. Lima, Perú.

Schulenberg, T.D., Stotz, D.S., Lane, D.F., O’Neill, J.P. \& Parker III, T.A. (2010). Aves de Perú. Princeton Univ. Press. Princeton, New Jersey.

Terborgh, J. (1971). Distribution on Environmental Gradients: Theory and Preliminary Interpretation of Distributional Patterns in the Avifauna of the Cordillera Vilcabamba, Peru. Ecology, 52 (1): 23-40.

Terborgh, J. (1977). Birds species diversity on an Andean elevational gradient. Ecology, 58: 1007-1019.

Walker, B. (2015). Field Guide to the Birds of Machu Picchu and the Cusco Region, Peru. Barcelona.

Walker, B., Stotz, D.F., Pequeño, T. \& Fitzpatrick, J.W. (2006). Birds of the Manu biosphere reserve. In: Patterson, D., stotz, D. \& solari, S. (Eds.), Mammals and birds of the Manu Biosphere Reserve, Peru. Fieldiana: Zoology, New Series, 110: 23-49.

Walker, B. \& Ricalde, D. (1998). Aves de Machupicchu y alrededores. Boletín de Lima, 58: 6979.

Walker, B. \& Stolz, D.F. (2017). Birds recorded within the Manu Biosphere Reserve, departments of Cusco and Madre de Dios, Peru. Publ. online. http://cochacashu.sandiegozooglobal.org/es/researchers/species-lists/

Young, K.R. (1992). Biogeography of the montane forest zone of the eastem slopes of Peru. En: Biogeografía, Ecología y Conservación del Bosque Montano en el Perú. Museo de Historia Natural UNMSM. 21. Lima. Perú.

Young, K.R. (1993). Biogeografía y conservación de los bosques montanos tropicales. En: Memoria X. CONABIOL. 1992. Lima, Perú. Pp. 57-62. 
Anexo 1. Lista de aves de bosques montanos en el departamento del Cusco.

\begin{tabular}{|c|c|c|c|c|c|c|c|c|c|c|c|}
\hline & Familia & Especie & Altitud & $\begin{array}{c}\text { Bosqu } \\
\mathrm{e} \\
\text { Enano } \\
(3800- \\
2500) \\
\end{array}$ & $\begin{array}{c}\text { Bosque } \\
\text { Nublad } \\
\text { o } \\
(2500- \\
1500) \\
\end{array}$ & \begin{tabular}{|c|} 
Bosque \\
Lluvios \\
o \\
$(1500-$ \\
$700)$ \\
\end{tabular} & Manu & $\begin{array}{c}\text { Machupicch } \\
\mathrm{u}\end{array}$ & Choquequirao & Yanatile & Marcapata \\
\hline 01 & Tinamidae & Crypturellus atrocapillus & $250-1000$ & & & $\mathrm{x}$ & $\mathrm{x}$ & & & & \\
\hline 02 & Tinamidae & Crypturellus cinereus & $250-1000$ & & & $\mathrm{x}$ & $\mathrm{x}$ & & & & \\
\hline 03 & Tinamidae & Crypturellus obsoletus & $2600-3055$ & $\mathrm{x}$ & & & $\mathrm{x}$ & $\mathrm{x}$ & & $\mathrm{x}$ & \\
\hline 04 & Tinamidae & Crypturellus soui & $250-1500$ & & $\mathrm{x}$ & $\mathrm{x}$ & $\mathrm{x}$ & & & & \\
\hline 05 & Tinamidae & Nothocercus nigrocapillus & $1600-3200$ & $\mathrm{x}$ & $\mathrm{x}$ & & $\mathrm{x}$ & & & & \\
\hline 06 & Tinamidae & Nothoprocta pentlandii & $2000-3600$ & $\mathrm{x}$ & $\mathrm{x}$ & & $\mathrm{x}$ & $\mathrm{x}$ & $\mathrm{x}$ & & \\
\hline 07 & Tinamidae & Nothoprocta taczanowskii & 3200 & $\mathrm{x}$ & & & $\mathrm{x}$ & $\mathrm{x}$ & & & \\
\hline 08 & Tinamidae & Tinamus guttatus & $250-1100$ & & & $\mathrm{x}$ & $\mathrm{x}$ & & & & \\
\hline 09 & Tinamidae & Tinamus osgoodi & $900-1400$ & & $\mathrm{x}$ & & $\mathrm{x}$ & & & & $\mathrm{x}$ \\
\hline 10 & Tinamidae & Tinamus tao & $250-1300$ & & & $\mathrm{x}$ & $\mathrm{x}$ & & & & \\
\hline 11 & Anatidae & Anas flavirostris & 3500 & $\mathrm{x}$ & & & $\mathrm{x}$ & & & & \\
\hline 12 & Anatidae & Anas georgica & 3200 & $\mathrm{x}$ & & & $\mathrm{x}$ & & & & \\
\hline 13 & Anatidae & Merganetta armata & $900-3500$ & & $\mathrm{x}$ & $\mathrm{x}$ & $\mathrm{x}$ & $\mathrm{X}$ & $\mathrm{x}$ & & \\
\hline 14 & Cracidae & Aburria aburri & $650-1600$ & & $\mathrm{x}$ & & $\mathrm{x}$ & & & & \\
\hline 15 & Cracidae & Chamaepetes goudotii & $1800-2800$ & & $\mathrm{x}$ & & $\mathrm{x}$ & $\mathrm{x}$ & $\mathrm{x}$ & & \\
\hline 16 & Cracidae & Ortalis guttata & $1500-2470$ & & & $\mathrm{x}$ & $\mathrm{x}$ & & & $\mathrm{x}$ & $\mathrm{x}$ \\
\hline 17 & Cracidae & Penelope jacquacu & $250-1500$ & & & $\mathrm{x}$ & $\mathrm{x}$ & & & & $\mathrm{x}$ \\
\hline 18 & Cracidae & Penelope montagnii & $1800-3500$ & $\mathrm{x}$ & $\mathrm{x}$ & & $\mathrm{x}$ & $\mathrm{x}$ & $\mathrm{x}$ & $\mathrm{x}$ & $\mathrm{x}$ \\
\hline 19 & Odontophoridae & Odontophorus speciosus & $1800-2600$ & & & $\mathrm{x}$ & $\mathrm{x}$ & $\mathrm{x}$ & & & $\mathrm{x}$ \\
\hline 20 & Odontophoridae & Odontophorus balliviani & $1800-3300$ & $\mathrm{x}$ & $\mathrm{x}$ & & $\mathrm{x}$ & $\mathrm{x}$ & & & $\mathrm{x}$ \\
\hline
\end{tabular}




\begin{tabular}{|c|c|c|c|c|c|c|c|c|c|c|c|}
\hline 21 & $\begin{array}{l}\text { Phalacrocoracida } \\
\text { e }\end{array}$ & Phalacrocorax brasilianus & $250-3600$ & & $\mathrm{x}$ & $\mathrm{x}$ & $\mathrm{x}$ & $\mathrm{x}$ & & & $\mathrm{X}$ \\
\hline 22 & Ardeidae & Bubulcus ibis & $250-1500$ & $\mathrm{x}$ & & & $\mathrm{x}$ & & & & \\
\hline 23 & Ardeidae & Tigrisoma fasciatum & $400-1600$ & & $\mathrm{x}$ & $\mathrm{x}$ & $\mathrm{x}$ & & & & $\mathrm{x}$ \\
\hline 24 & Thinocoridae & Thinocorus orbignyianus & $3000-4600$ & $\mathrm{x}$ & & & & & & & $\mathrm{x}$ \\
\hline 25 & Cathartidae & Cathartes aura & $900-2200$ & & $\mathrm{x}$ & $\mathrm{x}$ & $\mathrm{x}$ & & $\mathrm{x}$ & & $\mathrm{x}$ \\
\hline 26 & Cathartidae & Coragyps atratus & $250-1300$ & & & $\mathrm{x}$ & $\mathrm{x}$ & & & & $\mathrm{x}$ \\
\hline 27 & Cathartidae & Vultur gryphus & 3500 & $\mathrm{x}$ & & & $\mathrm{x}$ & & $\mathrm{x}$ & & \\
\hline 28 & Accipitridae & Accipiter collaris & $<2500$ & & $\mathrm{x}$ & & $\mathrm{x}$ & $\mathrm{x}$ & & & \\
\hline 29 & Accipitridae & Accipiter striatus & $900-3500$ & & $\mathrm{x}$ & $\mathrm{x}$ & $\mathrm{x}$ & & & & $\mathrm{x}$ \\
\hline 30 & Accipitridae & Accipiter ventralis & $1000-3500$ & & & $\mathrm{x}$ & & $\mathrm{x}$ & & & \\
\hline 31 & Accipitridae & Asturina nitida & $250-1200$ & & & $\mathrm{x}$ & $\mathrm{x}$ & & & & \\
\hline 32 & Accipitridae & Buteo albigula & $1800-3500$ & & $\mathrm{x}$ & $\mathrm{x}$ & $\mathrm{x}$ & $\mathrm{x}$ & $\mathrm{x}$ & & \\
\hline 33 & Accipitridae & Buteo brachyurus & 2600- 3055 & $\mathrm{x}$ & & & $\mathrm{x}$ & & & $\mathrm{x}$ & \\
\hline 34 & Accipitridae & Buteo platypterus & $<3000$ & $\mathrm{x}$ & & & $\mathrm{x}$ & $\mathrm{x}$ & & & \\
\hline 35 & Accipitridae & Chondrohierax uncinatus & $250-2000$ & & $\mathrm{x}$ & $\mathrm{x}$ & $\mathrm{x}$ & & & & \\
\hline 36 & Accipitridae & Circus cinereus & $2900-4000$ & $\mathrm{x}$ & & & $\mathrm{x}$ & & & & \\
\hline 37 & Accipitridae & Elanoides forficatus & $<2600$ & & $\mathrm{x}$ & $\mathrm{x}$ & $\mathrm{x}$ & $\mathrm{x}$ & & & \\
\hline 38 & Accipitridae & Geranoaetus melanoleucus & $2500-3800$ & $\mathrm{x}$ & $\mathrm{x}$ & & $\mathrm{x}$ & $\mathrm{x}$ & $\mathrm{x}$ & $\mathrm{x}$ & $\mathrm{x}$ \\
\hline 39 & Accipitridae & Geranoaetus polyosoma & $1800-4600$ & $\mathrm{x}$ & $\mathrm{x}$ & & $\mathrm{x}$ & $\mathrm{x}$ & & $\mathrm{x}$ & \\
\hline 40 & Accipitridae & Harpagus bidentatus & $250-1350$ & & & $\mathrm{x}$ & $\mathrm{x}$ & & & & \\
\hline 41 & Accipitridae & Harpyhaliaetus solitarius & $900-2100$ & & $\mathrm{x}$ & $\mathrm{x}$ & $\mathrm{x}$ & & $\mathrm{x}$ & & $\mathrm{X}$ \\
\hline 42 & Accipitridae & Ictinia plumbea & $250-1450$ & & & $\mathrm{x}$ & $\mathrm{x}$ & & & & \\
\hline 43 & Accipitridae & Leucopternis albicollis & $500-1500$ & & & $\mathrm{x}$ & $\mathrm{x}$ & & & & \\
\hline 44 & Accipitridae & Leucopternis kuhli & $1500-2470$ & & $\mathrm{x}$ & & & & & $\mathrm{x}$ & $\mathrm{x}$ \\
\hline 45 & Accipitridae & Parabuteo leucorrhous & $1400-3500$ & & $\mathrm{x}$ & & $\mathrm{x}$ & $\mathrm{x}$ & & & \\
\hline 46 & Accipitridae & Rupornis magnirostris & 15002470 & & & $\mathrm{x}$ & $\mathrm{x}$ & $\mathrm{x}$ & & $\mathrm{x}$ & $\mathrm{X}$ \\
\hline
\end{tabular}




\begin{tabular}{|c|c|c|c|c|c|c|c|c|c|c|c|}
\hline 47 & Accipitridae & Spizaetus isidori & $1800-3500$ & & $\mathrm{x}$ & $\mathrm{x}$ & $\mathrm{x}$ & $\mathrm{x}$ & $\mathrm{x}$ & $\mathrm{x}$ & \\
\hline 48 & Accipitridae & Spizaetus ornatus & $250-1100$ & & & $\mathrm{x}$ & $\mathrm{x}$ & & & & \\
\hline 49 & Accipitridae & Spizaetus tyrannus & $250-1100$ & & & $\mathrm{x}$ & $\mathrm{x}$ & & & & \\
\hline 50 & Accipitridae & Spizastur melanoleucus & $250-1300$ & & & $\mathrm{x}$ & $\mathrm{x}$ & & & & \\
\hline 51 & Falconidae & Eurypyga helias & $250-1600$ & & $\mathrm{x}$ & $\mathrm{x}$ & $\mathrm{x}$ & & & & \\
\hline 52 & Falconidae & Falco deiroleucus & $<2100$ & $\mathrm{x}$ & $\mathrm{x}$ & & & $\mathrm{x}$ & & & \\
\hline 53 & Falconidae & Falco femoralis & $2400-4300$ & $\mathrm{x}$ & $\mathrm{x}$ & & $\mathrm{x}$ & $\mathrm{x}$ & $\mathrm{x}$ & & \\
\hline 54 & Falconidae & Falco peregrinus & $350-3000$ & $\mathrm{x}$ & $\mathrm{x}$ & $\mathrm{x}$ & $\mathrm{x}$ & $\mathrm{x}$ & & & \\
\hline 55 & Falconidae & Falco rufigularis & $250-1500$ & & & $\mathrm{x}$ & $\mathrm{x}$ & & & & \\
\hline 56 & Falconidae & Falco sparverius & $2500-4500$ & $\mathrm{x}$ & $\mathrm{x}$ & & $\mathrm{x}$ & $\mathrm{x}$ & $\mathrm{x}$ & & $\mathrm{x}$ \\
\hline 57 & Falconidae & Himanopus mexicanus & $250-1000$ & $\mathrm{x}$ & & & $\mathrm{x}$ & & & & \\
\hline 58 & Falconidae & Ibycter americanus & $250-1250$ & & & $\mathrm{x}$ & $\mathrm{x}$ & & & & \\
\hline 59 & Falconidae & Micrastur ruficollis & $250-2000$ & & $\mathrm{x}$ & $\mathrm{x}$ & $\mathrm{x}$ & & & & \\
\hline 60 & Falconidae & Micrastur semitorquatus & $250-1200$ & & $\mathrm{x}$ & $\mathrm{x}$ & $\mathrm{x}$ & & & & \\
\hline 61 & Falconidae & Phalcoboenus megalopterus & $3500-5000$ & $\mathrm{x}$ & & & $\mathrm{x}$ & $\mathrm{x}$ & $\mathrm{x}$ & $\mathrm{x}$ & \\
\hline 62 & Rallidae & Pardirallus nigricans & $400-1050$ & & & $\mathrm{x}$ & & & & & $\mathrm{x}$ \\
\hline 63 & Scolopacidae & Actitis macularius & $250-3500$ & $\mathrm{x}$ & $\mathrm{x}$ & $\mathrm{x}$ & $\mathrm{x}$ & & & & \\
\hline 64 & Scolopacidae & Gallinago andina & $3000-4600$ & $\mathrm{x}$ & & & & $\mathrm{x}$ & & & \\
\hline 65 & Scolopacidae & Gallinago jamesoni & $2700-3500$ & $\mathrm{x}$ & & & $\mathrm{x}$ & $\mathrm{x}$ & & & \\
\hline 66 & Scolopacidae & Phalaropus tricolor & $250-3450$ & $\mathrm{x}$ & $\mathrm{x}$ & $\mathrm{x}$ & $\mathrm{x}$ & & & & \\
\hline 67 & Scolopacidae & Pluvialis dominica & $250-2000$ & & $\mathrm{x}$ & $\mathrm{x}$ & $\mathrm{x}$ & & & & \\
\hline 68 & Scolopacidae & Tringa melanoleuca & $250-2900$ & $\mathrm{x}$ & $\mathrm{x}$ & $\mathrm{x}$ & $\mathrm{x}$ & & & & \\
\hline 69 & Scolopacidae & Tringa solitaria & $250-3450$ & $\mathrm{x}$ & $\mathrm{x}$ & $\mathrm{x}$ & $\mathrm{x}$ & & & & \\
\hline 70 & Laridae & Chroicocephalus serranus & $3000-4400$ & $\mathrm{x}$ & & & & $\mathrm{x}$ & $\mathrm{x}$ & & \\
\hline 71 & Columbidae & Claravis mondetoura & $1800-3000$ & & $\mathrm{x}$ & & & $\mathrm{x}$ & & & \\
\hline 72 & Columbidae & Geotrygon montana & $<2000$ & & $\mathrm{x}$ & $\mathrm{x}$ & $\mathrm{x}$ & $\mathrm{x}$ & & & $\mathrm{x}$ \\
\hline 73 & Columbidae & Geotrygon saphirina & $600-1000$ & & & $\mathrm{x}$ & $\mathrm{x}$ & & & & \\
\hline
\end{tabular}




\begin{tabular}{|c|c|c|c|c|c|c|c|c|c|c|c|}
\hline 74 & Columbidae & Leptotila rufaxilla & $250-1250$ & & & $\mathrm{x}$ & $\mathrm{x}$ & & $\mathrm{x}$ & & $\mathrm{x}$ \\
\hline 75 & Columbidae & Leptotila verreauxi & $<2500$ & $\mathrm{x}$ & $\mathrm{x}$ & & & $\mathrm{x}$ & $\mathrm{x}$ & & \\
\hline 76 & Columbidae & Metriopelia ceciliae & $2500-4500$ & $\mathrm{x}$ & & & $\mathrm{x}$ & $\mathrm{x}$ & $\mathrm{x}$ & & \\
\hline 77 & Columbidae & Patagioenas fasciata & $2000-3000$ & & $\mathrm{x}$ & & $\mathrm{x}$ & $\mathrm{x}$ & $\mathrm{x}$ & & $\mathrm{x}$ \\
\hline 78 & Columbidae & Patagioenas plumbea & $<2000$ & & & $\mathrm{x}$ & $\mathrm{x}$ & $\mathrm{x}$ & $\mathrm{x}$ & & \\
\hline 79 & Columbidae & Patagioenas cayennensis & $250-900$ & & & $\mathrm{x}$ & $\mathrm{x}$ & $\mathrm{x}$ & & & \\
\hline 80 & Columbidae & Patagioenas maculosa & $3500-4000$ & $\mathrm{x}$ & & & $\mathrm{x}$ & $\mathrm{x}$ & $\mathrm{x}$ & & \\
\hline 81 & Columbidae & Patagioenas subvinacea & $250-1550$ & & $\mathrm{x}$ & $\mathrm{x}$ & $\mathrm{x}$ & $\mathrm{x}$ & & & \\
\hline 82 & Columbidae & Zenaida auriculata & $2500-4500$ & $\mathrm{x}$ & & & & $\mathrm{x}$ & $\mathrm{x}$ & & $\mathrm{x}$ \\
\hline 83 & Columbidae & Zentrygon frenata & $1800-3000$ & $\mathrm{x}$ & $\mathrm{x}$ & & & $\mathrm{x}$ & & $\mathrm{x}$ & \\
\hline 84 & Cuculidae & Piaya cayana & $900-2500$ & $\mathrm{x}$ & $\mathrm{x}$ & $\mathrm{x}$ & $\mathrm{x}$ & $\mathrm{x}$ & $\mathrm{x}$ & $\mathrm{x}$ & $\mathrm{x}$ \\
\hline 85 & Cuculidae & Coccyzus americanus & $250-1000$ & & $\mathrm{x}$ & $\mathrm{x}$ & $\mathrm{x}$ & & & & \\
\hline 86 & Cuculidae & Crotophaga ani & $900-2100$ & & $\mathrm{x}$ & $\mathrm{x}$ & $\mathrm{x}$ & $\mathrm{x}$ & $\mathrm{x}$ & $\mathrm{x}$ & \\
\hline 87 & Cuculidae & Coccyzus melacoryphus & $<2400$ & & $\mathrm{x}$ & & & $\mathrm{x}$ & & & \\
\hline 88 & Cuculidae & Coccyzus americanus & 2000 & & $\mathrm{x}$ & & & $\mathrm{x}$ & & & \\
\hline 89 & Tytonidae & Tyto alba & $1800-4000$ & $\mathrm{x}$ & $\mathrm{x}$ & $\mathrm{x}$ & $\mathrm{x}$ & $\mathrm{x}$ & & & \\
\hline 90 & Strigidae & Athene cunicularia & $1900-3500$ & $\mathrm{x}$ & $\mathrm{x}$ & & $\mathrm{x}$ & $\mathrm{x}$ & & & \\
\hline 91 & Strigidae & Bubo virginianus & $3400-3500$ & $\mathrm{x}$ & & & $\mathrm{x}$ & $\mathrm{x}$ & & & \\
\hline 92 & Strigidae & Ciccaba albitarsus & $1900-3500$ & & $\mathrm{x}$ & & $\mathrm{x}$ & $\mathrm{x}$ & $\mathrm{x}$ & & $\mathrm{x}$ \\
\hline 93 & Strigidae & Glaucidium bolivianum & $1800-3000$ & & $\mathrm{x}$ & $\mathrm{x}$ & $\mathrm{x}$ & $\mathrm{x}$ & & & \\
\hline 94 & Strigidae & Glaucidium brasilianum & $250-1000$ & & & $\mathrm{x}$ & $\mathrm{x}$ & & & & \\
\hline 95 & Strigidae & Glaucidium hardyi & $250-1150$ & & & $\mathrm{x}$ & $\mathrm{x}$ & & & & \\
\hline 96 & Strigidae & Glaucidium parkeri & 1000 & & $\mathrm{x}$ & $\mathrm{x}$ & $\mathrm{x}$ & & & & \\
\hline 97 & Strigidae & Glaucidium peruanum & $<3300$ & $\mathrm{x}$ & $\mathrm{x}$ & & & $\mathrm{x}$ & & & \\
\hline 98 & Strigidae & Megascops albogularis & $2000-3000$ & & $\mathrm{x}$ & & $\mathrm{x}$ & $\mathrm{x}$ & & & \\
\hline 99 & Strigidae & Megascops choliba & $<2400$ & $\mathrm{x}$ & $\mathrm{x}$ & & & $\mathrm{x}$ & & & \\
\hline 100 & Strigidae & Megascops guatemalae & $600-1600$ & & $\mathrm{x}$ & $\mathrm{x}$ & $\mathrm{x}$ & & & & \\
\hline
\end{tabular}




\begin{tabular}{|c|c|c|c|c|c|c|c|c|c|c|c|}
\hline 101 & Strigidae & Megascops ingens & $1500-3055$ & $\mathrm{x}$ & $\mathrm{x}$ & & $\mathrm{x}$ & & & $\mathrm{x}$ & \\
\hline 102 & Strigidae & Pulsatrix melanota & $<2300$ & & $\mathrm{X}$ & $\mathrm{x}$ & $\mathrm{x}$ & $\mathrm{x}$ & & & \\
\hline 103 & Steatornithidae & Steatornis caripensis & $1800-2000$ & & $\mathrm{x}$ & & $\mathrm{x}$ & $\mathrm{x}$ & & & \\
\hline 104 & Nictybiidae & Nyctibius maculosus & $1400-2800$ & & $\mathrm{x}$ & $\mathrm{x}$ & $\mathrm{x}$ & & & & \\
\hline 105 & Caprimulgidae & Caprimulgus nigrescens & $600-1200$ & & $\mathrm{x}$ & $\mathrm{x}$ & $\mathrm{x}$ & & & & \\
\hline 106 & Caprimulgidae & Hydropsalis torquata & $250-3055$ & $\mathrm{x}$ & $\mathrm{x}$ & $\mathrm{x}$ & & $\mathrm{x}$ & & $\mathrm{x}$ & \\
\hline 107 & Caprimulgidae & Lurocalis rufiventris & $1800-3300$ & & $\mathrm{x}$ & & $\mathrm{x}$ & $\mathrm{x}$ & & & \\
\hline 108 & Caprimulgidae & Nyctiphrynus ocellatus & $250-1000$ & & & $\mathrm{x}$ & $\mathrm{x}$ & & & & \\
\hline 109 & Caprimulgidae & Systellura longirostris & $2400-4200$ & & $\mathrm{x}$ & & & $\mathrm{x}$ & $\mathrm{x}$ & $\mathrm{x}$ & \\
\hline 110 & Caprimulgidae & Uropsalis lyra & $1800-2100$ & & $\mathrm{x}$ & $\mathrm{x}$ & $\mathrm{x}$ & $\mathrm{x}$ & & $\mathrm{x}$ & \\
\hline 111 & Caprimulgidae & Uropsalis segmentata & $2500-3600$ & $\mathrm{x}$ & $\mathrm{x}$ & & & $\mathrm{x}$ & & $\mathrm{x}$ & \\
\hline 112 & Apodidae & Aeronautes montivagus & $1800-2400$ & & $\mathrm{x}$ & $\mathrm{x}$ & $\mathrm{x}$ & $\mathrm{x}$ & $\mathrm{x}$ & & \\
\hline 113 & Apodidae & Chaetura brachyura & $250-1000$ & & & $\mathrm{x}$ & $\mathrm{x}$ & & & & \\
\hline 114 & Apodidae & Chaetura cinereiventris & $250-1450$ & & $\mathrm{x}$ & $\mathrm{x}$ & $\mathrm{x}$ & $\mathrm{x}$ & & & \\
\hline 115 & Apodidae & Chaetura egregia & $250-1600$ & & $\mathrm{x}$ & $\mathrm{x}$ & $\mathrm{x}$ & & & & \\
\hline 116 & Apodidae & Chaetura pelagica & $2300-4500$ & $\mathrm{x}$ & & & & $\mathrm{x}$ & & & \\
\hline 117 & Apodidae & Cypseloides cryptus & $600-1950$ & & $\mathrm{x}$ & & $\mathrm{x}$ & & & & \\
\hline 118 & Apodidae & Streptoprocne rutilus & $<3000$ & $\mathrm{x}$ & & & $\mathrm{x}$ & $\mathrm{x}$ & & $\mathrm{x}$ & \\
\hline 119 & Apodidae & Streptoprocne zonaris & $1800-4000$ & $\mathrm{x}$ & $\mathrm{x}$ & & $\mathrm{x}$ & $\mathrm{x}$ & $\mathrm{x}$ & & \\
\hline 120 & Trochilidae & Adelomyia melanogenys & $1000-2800$ & $\mathrm{x}$ & $\mathrm{x}$ & & $\mathrm{x}$ & $\mathrm{x}$ & $\mathrm{x}$ & $\mathrm{x}$ & \\
\hline 121 & Trochilidae & Aglaeactis castelnaudii & $2500-4100$ & $\mathrm{x}$ & & & $\mathrm{x}$ & $\mathrm{x}$ & $\mathrm{x}$ & & \\
\hline 122 & Trochilidae & Aglaiocercus kingi & $1200-2200$ & $\mathrm{x}$ & $\mathrm{x}$ & & $\mathrm{x}$ & $\mathrm{x}$ & & $\mathrm{x}$ & \\
\hline 123 & Trochilidae & Amazilia chionogaster & $1100-3400$ & $\mathrm{x}$ & & & $\mathrm{x}$ & $\mathrm{x}$ & $\mathrm{x}$ & $\mathrm{x}$ & \\
\hline 124 & Trochilidae & Amazilia lactea & $250-1400$ & & $\mathrm{x}$ & $\mathrm{x}$ & $\mathrm{x}$ & & & & \\
\hline 125 & Trochilidae & Amazilia viridicauda & $1500-2800$ & $\mathrm{x}$ & $\mathrm{x}$ & & $\mathrm{x}$ & $\mathrm{x}$ & $\mathrm{x}$ & $\mathrm{x}$ & $\mathrm{x}$ \\
\hline 126 & Trochilidae & Boissonneaua matthewsii & $1800-3300$ & $\mathrm{x}$ & $\mathrm{x}$ & & $\mathrm{x}$ & $\mathrm{x}$ & & $\mathrm{x}$ & $\mathrm{x}$ \\
\hline 127 & Trochilidae & Campylopterus largipennis & $250-1300$ & & $\mathrm{x}$ & $\mathrm{x}$ & $\mathrm{x}$ & & & & \\
\hline
\end{tabular}




\begin{tabular}{|c|c|c|c|c|c|c|c|c|c|c|c|}
\hline 128 & Trochilidae & Chaetocercus mulsant & $900-3000$ & & $\mathrm{x}$ & $\mathrm{x}$ & $\mathrm{x}$ & $\mathrm{x}$ & $\mathrm{x}$ & $\mathrm{x}$ & $\mathrm{x}$ \\
\hline 129 & Trochilidae & Chalcostigma olivaceum & $3500-4700$ & $\mathrm{x}$ & & & & $\mathrm{x}$ & & & \\
\hline 130 & Trochilidae & Chalcostigma ruficeps & $1800-3600$ & $\mathrm{x}$ & $\mathrm{x}$ & & $\mathrm{x}$ & $\mathrm{x}$ & & & \\
\hline 131 & Trochilidae & Chalcostigma stanleyi & $2800-4500$ & $\mathrm{x}$ & & & & $\mathrm{x}$ & $\mathrm{x}$ & & \\
\hline 132 & Trochilidae & Chlorestes notata & $500-900$ & & & $\mathrm{x}$ & $\mathrm{x}$ & & & & \\
\hline 133 & Trochilidae & Chlorostilbon mellisugus & $250-900$ & & & $\mathrm{x}$ & $\mathrm{x}$ & & & $\mathrm{x}$ & \\
\hline 134 & Trochilidae & Chrysuronia oenone & $250-1550$ & & $\mathrm{x}$ & $\mathrm{x}$ & $\mathrm{x}$ & & & & $\mathrm{x}$ \\
\hline 135 & Trochilidae & Coeligena coeligena & $1000-2200$ & & $\mathrm{x}$ & & $\mathrm{x}$ & $\mathrm{x}$ & $\mathrm{x}$ & & \\
\hline 136 & Trochilidae & Coeligena torquata & $1800-3000$ & $\mathrm{x}$ & & & $\mathrm{x}$ & $\mathrm{x}$ & & $\mathrm{x}$ & \\
\hline 137 & Trochilidae & Coeligena violifer & $1900-3900$ & $\mathrm{x}$ & $\mathrm{x}$ & & $\mathrm{x}$ & $\mathrm{x}$ & $\mathrm{x}$ & $\mathrm{x}$ & $\mathrm{x}$ \\
\hline 138 & Trochilidae & Colibri coruscans & $1800-4000$ & $\mathrm{x}$ & & $\mathrm{x}$ & $\mathrm{x}$ & $\mathrm{x}$ & $\mathrm{x}$ & $\mathrm{x}$ & $\mathrm{x}$ \\
\hline 139 & Trochilidae & Colibri delphinae & $800-1700$ & & $\mathrm{x}$ & $\mathrm{x}$ & $\mathrm{x}$ & & & & \\
\hline 140 & Trochilidae & Colibri thalassinus & $1000-3300$ & & $\mathrm{x}$ & $\mathrm{x}$ & $\mathrm{x}$ & $\mathrm{x}$ & $\mathrm{x}$ & $\mathrm{x}$ & \\
\hline 141 & Trochilidae & Discosura popelairii & $500-1500$ & & $\mathrm{x}$ & $\mathrm{x}$ & $\mathrm{x}$ & & & & \\
\hline 142 & Trochilidae & Doryfera johannae & $500-1000$ & & & $\mathrm{x}$ & $\mathrm{x}$ & & & & \\
\hline 143 & Trochilidae & Doryfera ludovicae & $800-2500$ & & $\mathrm{x}$ & $\mathrm{x}$ & $\mathrm{x}$ & $\mathrm{x}$ & & & $\mathrm{x}$ \\
\hline 144 & Trochilidae & Ensifera ensifera & $2400-3600$ & $\mathrm{x}$ & $\mathrm{x}$ & & $\mathrm{x}$ & $\mathrm{x}$ & $\mathrm{x}$ & $\mathrm{x}$ & $\mathrm{x}$ \\
\hline 145 & Trochilidae & Eriocnemis luciani & $2400-3500$ & $\mathrm{x}$ & $\mathrm{x}$ & & $\mathrm{x}$ & $\mathrm{x}$ & $\mathrm{x}$ & & $\mathrm{X}$ \\
\hline 146 & Trochilidae & Eupetonema macroura & $1000-1500$ & & & $\mathrm{x}$ & & $\mathrm{x}$ & & & \\
\hline 147 & Trochilidae & Eutoxeres condamini & $<2500$ & & $\mathrm{x}$ & $\mathrm{x}$ & $\mathrm{x}$ & $\mathrm{x}$ & & & $\mathrm{x}$ \\
\hline 148 & Trochilidae & Florisuga mellivora & $250-1200$ & & & $\mathrm{x}$ & $\mathrm{x}$ & & & & \\
\hline 149 & Trochilidae & Glaucis hirsutus & $250-1000$ & & & $\mathrm{x}$ & $\mathrm{x}$ & & & & \\
\hline 150 & Trochilidae & Haplophaedia assimilis & $1500-2500$ & & $\mathrm{x}$ & & $\mathrm{x}$ & $\mathrm{x}$ & & & \\
\hline 151 & Trochilidae & Heliangelus amethysticollis & $2000-3700$ & $\mathrm{x}$ & $\mathrm{x}$ & & $\mathrm{x}$ & $\mathrm{x}$ & $\mathrm{x}$ & $\mathrm{x}$ & \\
\hline 152 & Trochilidae & Helidoxia rubinoides & $1300-2200$ & & $\mathrm{x}$ & & $\mathrm{x}$ & & & & \\
\hline 153 & Trochilidae & Heliodoxa branickii & $650-1400$ & & $\mathrm{x}$ & $\mathrm{x}$ & $\mathrm{x}$ & & & & \\
\hline 154 & Trochilidae & Heliodoxa leadbeateri & $900-2000$ & & $\mathrm{x}$ & $\mathrm{x}$ & $\mathrm{x}$ & & & & \\
\hline
\end{tabular}




\begin{tabular}{|c|c|c|c|c|c|c|c|c|c|c|c|}
\hline 155 & Trochilidae & Heliodoxa rubinoides & 2000 & & $\mathrm{x}$ & $\mathrm{x}$ & & $\mathrm{x}$ & & & \\
\hline 156 & Trochilidae & Heliodoxa schreibersii & $600-1250$ & & & $\mathrm{x}$ & $\mathrm{x}$ & & & & \\
\hline 157 & Trochilidae & Heliothryx aurita & $250-1100$ & & & $\mathrm{x}$ & $\mathrm{x}$ & & & & \\
\hline 158 & Trochilidae & Klais guimeti & $500-1000$ & & & $\mathrm{x}$ & $\mathrm{x}$ & & & & $\mathrm{x}$ \\
\hline 159 & Trochilidae & Lafresnaya lafresnayi & $1800-3200$ & $\mathrm{x}$ & $\mathrm{x}$ & & $\mathrm{x}$ & $\mathrm{x}$ & $\mathrm{x}$ & & \\
\hline 160 & Trochilidae & Lesbia nuna & $2000-3800$ & $\mathrm{x}$ & $\mathrm{x}$ & & $\mathrm{x}$ & $\mathrm{x}$ & $\mathrm{x}$ & & \\
\hline 161 & Trochilidae & Lesbia victoriae & $2700-4000$ & $\mathrm{x}$ & & & & $\mathrm{x}$ & $\mathrm{x}$ & & \\
\hline 162 & Trochilidae & Lophornis delattrei & $500-1900$ & & & $\mathrm{x}$ & $\mathrm{x}$ & & & & \\
\hline 163 & Trochilidae & Metallura aeneocauda & $2800-3600$ & $\mathrm{x}$ & & & $\mathrm{x}$ & $\mathrm{x}$ & $\mathrm{x}$ & & \\
\hline 164 & Trochilidae & Metallura tyrianthina & $1900-3500$ & $\mathrm{x}$ & & & $\mathrm{x}$ & $\mathrm{x}$ & $\mathrm{x}$ & $\mathrm{x}$ & $\mathrm{x}$ \\
\hline 165 & Trochilidae & Ocreatus underwoodii & $1800-2400$ & $\mathrm{x}$ & $\mathrm{x}$ & & $\mathrm{x}$ & $\mathrm{x}$ & & $\mathrm{x}$ & $\mathrm{x}$ \\
\hline 166 & Trochilidae & Oreonympha nobilis & $2700-3900$ & $\mathrm{x}$ & & & & $\mathrm{x}$ & & & \\
\hline 167 & Trochilidae & Oreotrochilus estella & $3000-4500$ & $\mathrm{x}$ & & & $\mathrm{x}$ & $\mathrm{x}$ & & $\mathrm{x}$ & \\
\hline 168 & Trochilidae & Patagona gigas & $2500-4000$ & $\mathrm{x}$ & & & $\mathrm{x}$ & $\mathrm{x}$ & $\mathrm{x}$ & & \\
\hline 169 & Trochilidae & Phaethornis guy & $550-1600$ & & $\mathrm{x}$ & $\mathrm{x}$ & $\mathrm{x}$ & $\mathrm{x}$ & & & $\mathrm{x}$ \\
\hline 170 & Trochilidae & Phaethornis hispidus & $250-2470$ & & & $\mathrm{x}$ & $\mathrm{x}$ & & & $\mathrm{x}$ & \\
\hline 171 & Trochilidae & Phaethornis koepckeae & $500-1300$ & & & $\mathrm{x}$ & $\mathrm{x}$ & & & & \\
\hline 172 & Trochilidae & Phaethornis malaris & $250-1350$ & & & $\mathrm{x}$ & $\mathrm{x}$ & & & & \\
\hline 173 & Trochilidae & Phaethornis ruber & $250-1400$ & & $\mathrm{x}$ & $\mathrm{x}$ & $\mathrm{x}$ & & & & $\mathrm{x}$ \\
\hline 174 & Trochilidae & Phlogophilus harterti & $750-1500$ & & $\mathrm{x}$ & $\mathrm{x}$ & $\mathrm{x}$ & & & & $\mathrm{x}$ \\
\hline 175 & Trochilidae & Pterophanes cyanopterus & $2600-3700$ & $\mathrm{x}$ & & & $\mathrm{x}$ & $\mathrm{x}$ & $\mathrm{x}$ & $\mathrm{x}$ & $\mathrm{x}$ \\
\hline 176 & Trochilidae & $\begin{array}{l}\text { Ramphomicron } \\
\text { microrhynchum }\end{array}$ & $2500-3600$ & $\mathrm{x}$ & $\mathrm{x}$ & & $\mathrm{x}$ & $\mathrm{x}$ & $\mathrm{x}$ & & \\
\hline 177 & Trochilidae & Schistes geoffroyi & $800-1500$ & & & $\mathrm{x}$ & $\mathrm{x}$ & & & & \\
\hline 178 & Trochilidae & Taphrospilus hypostictus & $750-1500$ & & $\mathrm{x}$ & $\mathrm{x}$ & $\mathrm{x}$ & & & & $\mathrm{x}$ \\
\hline 179 & Trochilidae & Thalurania furcata & $250-2470$ & & $\mathrm{x}$ & $\mathrm{x}$ & $\mathrm{x}$ & & & $\mathrm{x}$ & $\mathrm{x}$ \\
\hline 180 & Trochilidae & Threnetes leucurus & $250-1200$ & & $\mathrm{x}$ & $\mathrm{x}$ & & & & & $\mathrm{x}$ \\
\hline
\end{tabular}




\begin{tabular}{|c|c|c|c|c|c|c|c|c|c|c|c|}
\hline 181 & Trochilidae & Threnetes niger & $250-1200$ & & & $\mathrm{x}$ & $\mathrm{x}$ & & & & \\
\hline 182 & Trogonidae & Pharomachrus antisianus & $1800-2300$ & & $\mathrm{x}$ & & $\mathrm{x}$ & & & & \\
\hline 183 & Trogonidae & Pharomachrus auriceps & $1800-2300$ & & $\mathrm{x}$ & & $\mathrm{x}$ & $\mathrm{x}$ & $\mathrm{x}$ & & \\
\hline 184 & Trogonidae & Trogon curucui & $250-1500$ & & $\mathrm{x}$ & $\mathrm{x}$ & $\mathrm{x}$ & & & & \\
\hline 185 & Trogonidae & Trogon personatus & $1800-3300$ & $\mathrm{x}$ & $\mathrm{x}$ & & $\mathrm{x}$ & $\mathrm{x}$ & $\mathrm{x}$ & $\mathrm{x}$ & $\mathrm{x}$ \\
\hline 186 & Alcedinidae & Chloroceryle amazona & $250-1300$ & & & $\mathrm{x}$ & $\mathrm{x}$ & & & & \\
\hline 187 & Alcedinidae & Chloroceryle americana & $250-1350$ & & & $\mathrm{x}$ & $\mathrm{x}$ & & & & \\
\hline 188 & Momotidae & Baryphthengus martii & 2000 & & $\mathrm{x}$ & $\mathrm{x}$ & $\mathrm{x}$ & $\mathrm{x}$ & & & $\mathrm{x}$ \\
\hline 189 & Momotidae & Momotus aequatorialis & $1500-2400$ & $\mathrm{x}$ & $\mathrm{x}$ & & $\mathrm{x}$ & $\mathrm{x}$ & & $\mathrm{x}$ & $\mathrm{x}$ \\
\hline 190 & Bucconidae & Bucco macrodactylus & $250-1000$ & & & $\mathrm{x}$ & $\mathrm{x}$ & & & & $\mathrm{x}$ \\
\hline 191 & Bucconidae & Malacoptila fulvogularis & $1800-2000$ & $\mathrm{x}$ & $\mathrm{x}$ & & $\mathrm{x}$ & $\mathrm{x}$ & & & $\mathrm{x}$ \\
\hline 192 & Bucconidae & Malacoptila semicincta & $250-1250$ & & & $\mathrm{x}$ & $\mathrm{x}$ & & & & \\
\hline 193 & Bucconidae & Micromonacha lanceolata & $500-1500$ & & $\mathrm{x}$ & $\mathrm{x}$ & $\mathrm{x}$ & & & & \\
\hline 194 & Bucconidae & Monasa flavirostris & $250-1250$ & & $\mathrm{x}$ & $\mathrm{x}$ & $\mathrm{x}$ & & & & \\
\hline 195 & Bucconidae & Nonnula ruficapilla & $250-1250$ & & $\mathrm{x}$ & $\mathrm{x}$ & $\mathrm{x}$ & & & & \\
\hline 196 & Bucconidae & Nystalus striolatus & $250-1200$ & & & $\mathrm{x}$ & $\mathrm{x}$ & & & & \\
\hline 197 & Galbulidae & Galbula cyanescens & $1500-2470$ & & & $\mathrm{x}$ & $\mathrm{x}$ & & & $\mathrm{x}$ & $\mathrm{x}$ \\
\hline 198 & Capitonidae & Capito auratus & $250-1350$ & & & $\mathrm{x}$ & $\mathrm{x}$ & & & & \\
\hline 199 & Capitonidae & Eubucco richardsoni & $250-1000$ & & & $\mathrm{x}$ & $\mathrm{x}$ & & & & $\mathrm{x}$ \\
\hline 200 & Capitonidae & Eubucco versicolor & $<2100$ & & $\mathrm{x}$ & & $\mathrm{x}$ & $\mathrm{x}$ & & & $\mathrm{x}$ \\
\hline 201 & Capitonidae & Eubucco richardsoni & & $\mathrm{x}$ & $\mathrm{x}$ & & & & & & $\mathrm{x}$ \\
\hline 202 & Ramphastidae & Andigena hypoglauca & $2300-3500$ & $\mathrm{x}$ & $\mathrm{x}$ & & $\mathrm{x}$ & $\mathrm{x}$ & $\mathrm{x}$ & & \\
\hline 203 & Ramphastidae & $\begin{array}{l}\text { Aulacorhynchus (prasinus) } \\
\text { atrogularis }\end{array}$ & $<2000$ & & $\mathrm{x}$ & & $\mathrm{X}$ & $\mathrm{x}$ & & & $\mathrm{X}$ \\
\hline 204 & Ramphastidae & $\begin{array}{l}\text { Aulacorhynchus } \\
\text { coeruleicinctis }\end{array}$ & $1800-2500$ & & $\mathrm{x}$ & & $\mathrm{x}$ & $\mathrm{x}$ & & & $\mathrm{X}$ \\
\hline 205 & Ramphastidae & Aulacorhynchus derbianus & $800-2100$ & & $\mathrm{x}$ & $\mathrm{x}$ & $\mathrm{x}$ & & & & $\mathrm{x}$ \\
\hline 206 & Ramphastidae & Pteroglossus azara & $250-1200$ & & & $\mathrm{x}$ & $\mathrm{x}$ & & & & \\
\hline
\end{tabular}




\begin{tabular}{|c|c|c|c|c|c|c|c|c|c|c|c|}
\hline 207 & Ramphastidae & Pteroglossus castanotis & $1500-3055$ & & $\mathrm{x}$ & $\mathrm{x}$ & $\mathrm{x}$ & & & $\mathrm{x}$ & $\mathrm{X}$ \\
\hline 208 & Picidae & Campephilus haematogaster & $<2300$ & & $\mathrm{X}$ & & $\mathrm{X}$ & $\mathrm{x}$ & & & \\
\hline 209 & Picidae & Campephilus melanoleucos & $250-1400$ & & & $\mathrm{x}$ & $\mathrm{x}$ & $\mathrm{x}$ & & & \\
\hline 210 & Picidae & Celeus grammicus & $250-1200$ & & & $\mathrm{x}$ & $\mathrm{x}$ & & & & \\
\hline 211 & Picidae & Colaptes punctigula & $<1150$ & & & $\mathrm{x}$ & & & & & $\mathrm{x}$ \\
\hline 212 & Picidae & Colaptes rupicola & $2500-4500$ & $\mathrm{x}$ & & & $\mathrm{x}$ & $\mathrm{x}$ & $\mathrm{x}$ & & \\
\hline 213 & Picidae & Dryocopus lineatus & $250-1550$ & & & $\mathrm{x}$ & $\mathrm{x}$ & & & & $\mathrm{x}$ \\
\hline 214 & Picidae & Melanerpes cruentatus & $250-1200$ & & & $\mathrm{x}$ & $\mathrm{x}$ & & & $\mathrm{x}$ & $\mathrm{x}$ \\
\hline 215 & Picidae & Piculus rivolii & $1000-3400$ & $\mathrm{x}$ & $\mathrm{x}$ & & $\mathrm{x}$ & $\mathrm{x}$ & $\mathrm{x}$ & & $\mathrm{x}$ \\
\hline 216 & Picidae & Colaptes rubiginosus & $<2400$ & & $\mathrm{x}$ & & $\mathrm{x}$ & $\mathrm{x}$ & $\mathrm{x}$ & $\mathrm{x}$ & $\mathrm{x}$ \\
\hline 217 & Picidae & Picummus subtilis & $450-1100$ & & & $\mathrm{x}$ & $\mathrm{x}$ & & & & $\mathrm{x}$ \\
\hline 218 & Picidae & Picumnus aurifrons & $250-1250$ & & & $\mathrm{x}$ & $\mathrm{x}$ & & & & \\
\hline 219 & Picidae & Picumnus dorbygnianus & $800-2400$ & & $\mathrm{x}$ & & $\mathrm{x}$ & $\mathrm{x}$ & & $\mathrm{x}$ & \\
\hline 220 & Picidae & Picumnus rufiventris & $250-1250$ & & & $\mathrm{x}$ & $\mathrm{x}$ & & & & \\
\hline 221 & Picidae & Veniliornis affinis & $250-1400$ & & & $\mathrm{x}$ & $\mathrm{x}$ & & & & $\mathrm{x}$ \\
\hline 222 & Picidae & Veniliornis dignus & $1500-2100$ & & $\mathrm{x}$ & & & & & & $\mathrm{x}$ \\
\hline 223 & Picidae & Veniliornis nigriceps & $2400-3500$ & $\mathrm{x}$ & & & $\mathrm{x}$ & $\mathrm{x}$ & $\mathrm{x}$ & & \\
\hline 224 & Picidae & Veniliornis passerinus & $250-1000$ & & & $\mathrm{x}$ & $\mathrm{x}$ & & & & $\mathrm{x}$ \\
\hline 225 & Psittacidae & Amazona farinosa & $250-1200$ & & & $\mathrm{x}$ & $\mathrm{x}$ & & & & \\
\hline 226 & Psittacidae & Amazona mercenaria & $1800-3500$ & $\mathrm{x}$ & & & $\mathrm{x}$ & $\mathrm{x}$ & & $\mathrm{x}$ & $\mathrm{X}$ \\
\hline 227 & Psittacidae & Ara militaris & $600-1500$ & & & $\mathrm{x}$ & $\mathrm{x}$ & & & & \\
\hline 228 & Psittacidae & Ara severus & $250-1200$ & & & $\mathrm{x}$ & $\mathrm{x}$ & & & & \\
\hline 229 & Psittacidae & Bolborhynchus lineola & $<3300$ & $\mathrm{x}$ & & & $\mathrm{x}$ & $\mathrm{x}$ & $\mathrm{x}$ & & \\
\hline 230 & Psittacidae & Bolborhynchus orbygnesius & $<3300$ & $\mathrm{x}$ & & & $\mathrm{x}$ & $\mathrm{x}$ & $\mathrm{x}$ & & \\
\hline 231 & Psittacidae & Leptosittaca branickii & $2700-3500$ & $\mathrm{x}$ & & & $\mathrm{x}$ & $\mathrm{x}$ & & & \\
\hline 232 & Psittacidae & Nannopsittaca dachilleae & $250-1050$ & & & $\mathrm{x}$ & $\mathrm{x}$ & & & & \\
\hline 233 & Psittacidae & Pionus menstruus & $250-1250$ & & & $\mathrm{x}$ & $\mathrm{x}$ & $\mathrm{x}$ & & & $\mathrm{x}$ \\
\hline
\end{tabular}




\begin{tabular}{|c|c|c|c|c|c|c|c|c|c|c|c|}
\hline 234 & Psittacidae & Pionus tumultuosus & $1800-3300$ & $\mathrm{x}$ & & & $\mathrm{x}$ & $\mathrm{x}$ & $\mathrm{x}$ & & \\
\hline 235 & Psittacidae & Propyrrhura couloni & $250-1300$ & & & $\mathrm{x}$ & $\mathrm{x}$ & & & & \\
\hline 236 & Psittacidae & Psittacara leucophthalmus & $1500-3055$ & $\mathrm{x}$ & $\mathrm{x}$ & & $\mathrm{x}$ & & & $\mathrm{x}$ & \\
\hline 237 & Psittacidae & Psittacara mitrata & $1800-3300$ & $\mathrm{x}$ & & & $\mathrm{x}$ & $\mathrm{x}$ & $\mathrm{x}$ & $\mathrm{x}$ & \\
\hline 238 & Psittacidae & Pyrrhura roseifrons & $250-1000$ & & & $\mathrm{x}$ & $\mathrm{x}$ & & & & \\
\hline 239 & Psittacidae & Pyrrhura rupicola & $250-1100$ & & & $\mathrm{x}$ & $\mathrm{x}$ & & & & \\
\hline 240 & Thamnophilidae & Cercomacra manu & $250-1350$ & & & $\mathrm{x}$ & $\mathrm{x}$ & & & & \\
\hline 241 & Thamnophilidae & Cercomacra serva & $250-1550$ & & & $\mathrm{x}$ & $\mathrm{x}$ & & & & \\
\hline 242 & Thamnophilidae & Cymbilaimus sanctaemariae & $250-1450$ & & & $\mathrm{x}$ & $\mathrm{x}$ & & & & \\
\hline 243 & Thamnophilidae & Drymophila caudata & 2100 & & $\mathrm{x}$ & & $\mathrm{x}$ & & & & \\
\hline 244 & Thamnophilidae & Drymophila striaticeps & $<2500$ & & $\mathrm{x}$ & & & $\mathrm{x}$ & & & \\
\hline 245 & Thamnophilidae & Dysithamnus mentalis & $600-1600$ & & & $\mathrm{x}$ & $\mathrm{x}$ & & & & \\
\hline 246 & Thamnophilidae & Frederickena unduligera & $250-1050$ & & & $\mathrm{x}$ & $\mathrm{x}$ & & & & \\
\hline 247 & Thamnophilidae & Herpsilochmus axillaris & $750-1600$ & & & $\mathrm{x}$ & $\mathrm{x}$ & & & & \\
\hline 248 & Thamnophilidae & Hylophylax naevius & $250-1200$ & & & $\mathrm{x}$ & $\mathrm{x}$ & & & & \\
\hline 249 & Thamnophilidae & Hypocnemis peruviana & $1500-2470$ & & $\mathrm{x}$ & & & & & $\mathrm{x}$ & $\mathrm{x}$ \\
\hline 250 & Thamnophilidae & Hypocnemis subflava & $250-1600$ & & & $\mathrm{x}$ & $\mathrm{x}$ & & & & \\
\hline 251 & Thamnophilidae & Microrhopias quixensis & $250-1350$ & & & $\mathrm{x}$ & $\mathrm{x}$ & & & & \\
\hline 252 & Thamnophilidae & Myrmeciza atrothorax & $250-1000$ & & & $\mathrm{x}$ & $\mathrm{x}$ & & & & \\
\hline 253 & Thamnophilidae & Myrmeciza fortis & $250-1300$ & & & $\mathrm{x}$ & $\mathrm{x}$ & & & & \\
\hline 254 & Thamnophilidae & Myrmeciza hemimelaena & $250-1550$ & & & $\mathrm{x}$ & $\mathrm{x}$ & & & & \\
\hline 255 & Thamnophilidae & Myrmoborus leucophrys & $250-1350$ & & & $\mathrm{x}$ & $\mathrm{x}$ & & & & \\
\hline 256 & Thamnophilidae & Myrmotherula axillaris & $250-1400$ & & & $\mathrm{x}$ & $\mathrm{x}$ & & & & \\
\hline 257 & Thamnophilidae & Myrmotherula brachyura & $250-1300$ & & & $\mathrm{x}$ & $\mathrm{x}$ & & & & \\
\hline 258 & Thamnophilidae & Myrmotherula longicauda & $500-1550$ & & & $\mathrm{x}$ & $\mathrm{x}$ & & & & $\mathrm{x}$ \\
\hline 259 & Thamnophilidae & Myrmotherula menestriesii & $250-1100$ & & & $\mathrm{x}$ & $\mathrm{x}$ & & & & $\mathrm{x}$ \\
\hline 260 & Thamnophilidae & Myrmotherula ornata & $250-1500$ & & & $\mathrm{x}$ & $\mathrm{x}$ & & & & \\
\hline
\end{tabular}




\begin{tabular}{|c|c|c|c|c|c|c|c|c|c|c|c|}
\hline 261 & Thamnophilidae & Myrmotherula schisticolor & $1100-1950$ & & $\mathrm{x}$ & $\mathrm{x}$ & $\mathrm{x}$ & & & & \\
\hline 262 & Thamnophilidae & Myrmotherula spodionota & $700-1350$ & & & $\mathrm{x}$ & $\mathrm{x}$ & & & & \\
\hline 263 & Thamnophilidae & Percnostola lophotes & $250-1350$ & & & $\mathrm{x}$ & $\mathrm{x}$ & & & & \\
\hline 264 & Thamnophilidae & Phlegopsis nigromaculata & $250-1250$ & & & $\mathrm{x}$ & $\mathrm{x}$ & & & & \\
\hline 265 & Thamnophilidae & Pygiptila stellaris & $250-600$ & & & $\mathrm{x}$ & $\mathrm{x}$ & & & & \\
\hline 266 & Thamnophilidae & Pyriglena leuconota & $500-1650$ & & & $\mathrm{x}$ & $\mathrm{x}$ & & & & \\
\hline 267 & Thamnophilidae & $\begin{array}{l}\text { Schistocichla } \\
\text { (Percnostola)brunneiceps }\end{array}$ & $500-1400$ & & & $\mathrm{x}$ & $\mathrm{x}$ & & & & \\
\hline 268 & Thamnophilidae & Taraba major & $250-1500$ & & & $\mathrm{x}$ & $\mathrm{x}$ & & & & \\
\hline 269 & Thamnophilidae & Terenura callinota & $750-1000$ & & & $\mathrm{x}$ & $\mathrm{x}$ & & & & \\
\hline 270 & Thamnophilidae & Terenura sharpei & $1000-1450$ & & & $\mathrm{x}$ & $\mathrm{x}$ & & & & \\
\hline 271 & Thamnophilidae & Thammophilus schistaceus & $250-1400$ & & & $\mathrm{x}$ & $\mathrm{x}$ & & & & $\mathrm{x}$ \\
\hline 272 & Thamnophilidae & Thamnistes anabatinus & $700-1500$ & & & $\mathrm{x}$ & $\mathrm{x}$ & & & & \\
\hline 273 & Thamnophilidae & Thamnomanes schistogynus & $250-1400$ & & & $\mathrm{x}$ & $\mathrm{x}$ & & & & \\
\hline 274 & Thamnophilidae & Thamnophilus caerulescens & $<2500$ & & $\mathrm{x}$ & $\mathrm{x}$ & $\mathrm{x}$ & $\mathrm{x}$ & & & \\
\hline 275 & Thamnophilidae & Thamnophilus doliatus & $1707-3055$ & & $\mathrm{x}$ & & $\mathrm{x}$ & & & $\mathrm{x}$ & \\
\hline 276 & Thamnophilidae & Thamnophilus palliatus & $500-1600$ & & & $\mathrm{x}$ & $\mathrm{x}$ & & & & $\mathrm{x}$ \\
\hline 277 & Thamnophilidae & Thamnophilus unicolor & $1250-2000$ & & $\mathrm{x}$ & $\mathrm{x}$ & $\mathrm{x}$ & & & & \\
\hline 278 & Conopophagidae & Conophaga castaneiceps & $1200-2000$ & & $\mathrm{x}$ & $\mathrm{x}$ & $\mathrm{x}$ & & & & \\
\hline 279 & Conopophagidae & Conopophaga ardesiaca & $850-1600$ & & & $\mathrm{x}$ & $\mathrm{x}$ & & & & \\
\hline 280 & Grallariidae & Grallaria albigula & $1150-2100$ & & $\mathrm{x}$ & & $\mathrm{x}$ & & & & \\
\hline 281 & Grallariidae & Grallaria blakei & $<1000$ & & & $\mathrm{x}$ & & & & & $\mathrm{x}$ \\
\hline 282 & Grallaridae & Grallaria erythroleuca & $2000-3000$ & $\mathrm{x}$ & & & $\mathrm{x}$ & $\mathrm{x}$ & $\mathrm{x}$ & $\mathrm{x}$ & \\
\hline 283 & Grallaridae & Grallaria guatimalensis & $<2000$ & & $\mathrm{x}$ & & $\mathrm{x}$ & $\mathrm{x}$ & & & \\
\hline 284 & Grallaridae & Grallaria rufula occabambae & $2600-3600$ & $\mathrm{x}$ & & & $\mathrm{x}$ & $\mathrm{x}$ & $\mathrm{x}$ & & \\
\hline 285 & Grallaridae & Grallaria squamigera & $2000-3500$ & $\mathrm{x}$ & & & $\mathrm{x}$ & $\mathrm{x}$ & $\mathrm{x}$ & & \\
\hline 286 & Grallaridae & $\begin{array}{l}\text { Grallaricula ferrugineipectus } \\
\text { leymebambae }\end{array}$ & $2000-3300$ & $\mathrm{x}$ & & & $\mathrm{X}$ & $\mathrm{x}$ & & & \\
\hline
\end{tabular}




\begin{tabular}{|c|c|c|c|c|c|c|c|c|c|c|c|}
\hline 287 & Grallariidae & Grallaricula flavirostris & $800-2200$ & & $\mathrm{x}$ & $\mathrm{x}$ & $\mathrm{x}$ & & & & \\
\hline 288 & Rhinocryptidae & Scytalopus simonsii & $2900-4300$ & $\mathrm{x}$ & & & $\mathrm{x}$ & $\mathrm{x}$ & & & \\
\hline 289 & Rhinocryptidae & Scytalopus atratus & $1000-2200$ & & $\mathrm{x}$ & $\mathrm{x}$ & $\mathrm{x}$ & & & & \\
\hline 290 & Rhinocryptidae & Scytalopus parvirostris & $2100-3600$ & $\mathrm{x}$ & & & & $\mathrm{x}$ & $\mathrm{x}$ & & \\
\hline 291 & Rhinocryptidae & Scytalopus parvirostris & $2100-3450$ & $\mathrm{x}$ & $\mathrm{x}$ & & $\mathrm{x}$ & & & & \\
\hline 292 & Rhinocryptidae & Scytalopus schulenbergi & $2750-3400$ & $\mathrm{x}$ & & & $\mathrm{x}$ & $\mathrm{x}$ & & & \\
\hline 293 & Rhinocryptidae & Scytalopus urubambae & $3500-4100$ & $\mathrm{x}$ & & & & $\mathrm{x}$ & & & \\
\hline 294 & Formicariidae & Chamaeza campanisona & $900-1500$ & & & $\mathrm{x}$ & $\mathrm{x}$ & & & & $\mathrm{x}$ \\
\hline 295 & Formicariidae & Chamaeza mollissima & $2000-3000$ & $\mathrm{x}$ & & & $\mathrm{x}$ & $\mathrm{x}$ & & & \\
\hline 296 & Formicariidae & Formicarius rufipectus & $<2400$ & $\mathrm{x}$ & & & $\mathrm{x}$ & $\mathrm{x}$ & & $\mathrm{x}$ & \\
\hline 297 & Furnariidae & Anabacerthia striaticollis & $1000-2000$ & & $\mathrm{x}$ & $\mathrm{x}$ & $\mathrm{x}$ & $\mathrm{x}$ & & & \\
\hline 298 & Furnariidae & Anabazenops dorsalis & $250-1350$ & & & $\mathrm{x}$ & $\mathrm{x}$ & & & & \\
\hline 299 & Furnariidae & Ancistrops strigilatus & $250-1100$ & & & $\mathrm{x}$ & $\mathrm{x}$ & & & & \\
\hline 300 & Furnariidae & Asthenes helleri & $2700-3700$ & $\mathrm{x}$ & & & & $\mathrm{x}$ & & & \\
\hline 301 & Furnariidae & Asthenes humilis & $3700-4800$ & $\mathrm{x}$ & & & $\mathrm{x}$ & & $\mathrm{x}$ & & \\
\hline 302 & Furnariidae & Asthenes modesta & $3600-4600$ & $\mathrm{X}$ & & & & & $\mathrm{x}$ & & \\
\hline 303 & Furnariidae & Asthenes ottonis & $2900-4000$ & $\mathrm{x}$ & & & & & $\mathrm{x}$ & & \\
\hline 304 & Furnariidae & Asthenes urubambensis & $3100-3800$ & $\mathrm{x}$ & & & $\mathrm{x}$ & $\mathrm{x}$ & & & \\
\hline 305 & Furnariidae & Asthenes virgata & $3350-4500$ & $\mathrm{x}$ & & & & $\mathrm{x}$ & & & \\
\hline 306 & Furnariidae & Automolus ochrolaemus & $250-1400$ & & & $\mathrm{x}$ & $\mathrm{x}$ & & & & \\
\hline 307 & Furnariidae & Automolus rubiginosus & $250-1400$ & & & $\mathrm{x}$ & $\mathrm{x}$ & & & & \\
\hline 308 & Furnariidae & Berlepschia rikeri & $250-600$ & & & $\mathrm{x}$ & $\mathrm{x}$ & & & & \\
\hline 309 & Furnariidae & Campylorhamphus pucherani & $2100-3000$ & $\mathrm{x}$ & $\mathrm{x}$ & & $\mathrm{x}$ & & & & \\
\hline 310 & Furnariidae & $\begin{array}{l}\text { Campylorhamphus } \\
\text { trochilirostris }\end{array}$ & $250-1500$ & & & $\mathrm{x}$ & $\mathrm{X}$ & & & & \\
\hline 311 & Furnariidae & Cinclodes albiventris & $2750-4800$ & $\mathrm{x}$ & $\mathrm{x}$ & & & $\mathrm{x}$ & $\mathrm{x}$ & & $\mathrm{x}$ \\
\hline 312 & Furnariidae & Cinclodes atacamensis & $2800-4800$ & $\mathrm{x}$ & $\mathrm{x}$ & & & $\mathrm{x}$ & $\mathrm{x}$ & & \\
\hline
\end{tabular}




\begin{tabular}{|c|c|c|c|c|c|c|c|c|c|c|c|}
\hline 313 & Furnariidae & Cranioleuca albicapilla & $2500-3600$ & $\mathrm{x}$ & $\mathrm{x}$ & & & $\mathrm{x}$ & $\mathrm{x}$ & $\mathrm{x}$ & \\
\hline 314 & Furnariidae & Cranioleuca curtata & $700-1600$ & & $\mathrm{x}$ & $\mathrm{x}$ & $\mathrm{x}$ & & & & \\
\hline 315 & Furnariidae & Cranioleuca marcapatae & 15003500 & $\mathrm{x}$ & $\mathrm{x}$ & & & $\mathrm{x}$ & & $\mathrm{x}$ & $\mathrm{x}$ \\
\hline 316 & Furnariidae & Deconychura longicauda & $250-1250$ & & & $\mathrm{x}$ & $\mathrm{x}$ & & & & \\
\hline 317 & Furnariidae & Dendrexetastes rufigula & $250-1100$ & & & $\mathrm{x}$ & $\mathrm{x}$ & & & & \\
\hline 318 & Furnariidae & Dendrocincla tyrannina & $2100-3150$ & $\mathrm{x}$ & $\mathrm{x}$ & & $\mathrm{x}$ & & & & \\
\hline 319 & Furnariidae & Dendrocolaptes picumnus & $250-1350$ & & & $\mathrm{x}$ & $\mathrm{x}$ & & & & \\
\hline 320 & Furnariidae & Drymotoxeres pucheranii & $3600-5000$ & $\mathrm{x}$ & & & & $\mathrm{x}$ & & & \\
\hline 321 & Furnariidae & Geositta cunicularia & $3100-4800$ & $\mathrm{x}$ & & & & $\mathrm{x}$ & $\mathrm{x}$ & & \\
\hline 322 & Furnariidae & Geositta tenuirostris & $3100-3500$ & $\mathrm{x}$ & & & $\mathrm{x}$ & $\mathrm{x}$ & & & \\
\hline 323 & Furnariidae & Glyphorhynchus spirurus & $250-1400$ & & & $\mathrm{x}$ & $\mathrm{x}$ & & & & $\mathrm{x}$ \\
\hline 324 & Furnariidae & Hyloctistes subulatus & $250-1300$ & & & $\mathrm{x}$ & $\mathrm{x}$ & & & & \\
\hline 325 & Furnariidae & Lepidocolaptes lacrymiger & $2000-3200$ & $\mathrm{x}$ & $\mathrm{x}$ & & & $\mathrm{x}$ & & $\mathrm{x}$ & \\
\hline 326 & Furnariidae & Leptasthenura andicola & 3500 & $\mathrm{x}$ & & & $\mathrm{x}$ & & & & \\
\hline 327 & Furnariidae & Lochmias nematura & $500-2300$ & & $\mathrm{x}$ & $\mathrm{x}$ & $\mathrm{x}$ & & & & \\
\hline 328 & Furnariidae & Margarornis squamiger & $2100-3700$ & $\mathrm{x}$ & $\mathrm{x}$ & & $\mathrm{x}$ & $\mathrm{x}$ & $\mathrm{x}$ & $\mathrm{x}$ & $\mathrm{x}$ \\
\hline 329 & Furnariidae & Metopothrix aurantiacus & $250-1100$ & & & $\mathrm{x}$ & $\mathrm{x}$ & & & & \\
\hline 330 & Furnariidae & $\begin{array}{l}\text { Philydor erythrocercum } \\
\text { (ochrogaster) }\end{array}$ & $750-1600$ & & $\mathrm{x}$ & $\mathrm{x}$ & $\mathrm{x}$ & & & & \\
\hline 331 & Furnariidae & Phleocryptes melanops & $3200-4300$ & $\mathrm{x}$ & & & & & $\mathrm{x}$ & & \\
\hline 332 & Furnariidae & Premnoplex brunnescens & $900-2800$ & & $\mathrm{x}$ & $\mathrm{x}$ & $\mathrm{x}$ & $\mathrm{x}$ & & & \\
\hline 333 & Furnariidae & Premnornis guttuliger & $1300-2500$ & & $\mathrm{x}$ & $\mathrm{x}$ & $\mathrm{x}$ & $\mathrm{x}$ & & & \\
\hline 334 & Furnariidae & Pseudocolaptes boissonneautii & $2000-3500$ & $\mathrm{x}$ & $\mathrm{x}$ & & $\mathrm{x}$ & $\mathrm{x}$ & & $\mathrm{x}$ & $\mathrm{x}$ \\
\hline 335 & Furnariidae & Schizoeaca helleri & $2700-3500$ & $\mathrm{x}$ & & & $\mathrm{x}$ & & & & \\
\hline 336 & Furnariidae & Sclerurus albigularis & $350-1500$ & & & $\mathrm{x}$ & $\mathrm{x}$ & & & $\mathrm{x}$ & \\
\hline 337 & Furnariidae & Simoxenops ucayalae & $250-1300$ & & & $\mathrm{x}$ & $\mathrm{x}$ & & & & \\
\hline 338 & Furnariidae & Sittasomus griseicapillus & $250-1200$ & & & $\mathrm{x}$ & $\mathrm{x}$ & & & & \\
\hline
\end{tabular}




\begin{tabular}{|c|c|c|c|c|c|c|c|c|c|c|c|}
\hline 339 & Furnariidae & Synallaxis azarae & $1000-3000$ & $\mathrm{x}$ & $\mathrm{x}$ & $\mathrm{x}$ & $\mathrm{x}$ & $\mathrm{x}$ & $\mathrm{x}$ & $\mathrm{x}$ & $\mathrm{x}$ \\
\hline 340 & Furnariidae & Synallaxis cabanisi & $250-1500$ & & & $\mathrm{x}$ & $\mathrm{x}$ & & & & \\
\hline 341 & Furnariidae & Synallaxis gujanensis & $250-1300$ & & & $\mathrm{x}$ & $\mathrm{x}$ & & & & \\
\hline 342 & Furnariidae & Syndactyla rufosuperciliata & $1000-1950$ & & $\mathrm{x}$ & $\mathrm{x}$ & $\mathrm{x}$ & & & & \\
\hline 343 & Furnariidae & Thamnophilus caerulescens & $1000-3000$ & $\mathrm{x}$ & $\mathrm{x}$ & $\mathrm{x}$ & & & $\mathrm{x}$ & & \\
\hline 344 & Furnariidae & Thripadectes holostictus & $1500-2500$ & & $\mathrm{x}$ & & $\mathrm{x}$ & $\mathrm{x}$ & & & $\mathrm{x}$ \\
\hline 345 & Furnariidae & Thripadectes melanorhynchus & $900-1600$ & & & $\mathrm{x}$ & $\mathrm{x}$ & & & & \\
\hline 346 & Furnariidae & Thripadectes scrutator & $2100-3500$ & $\mathrm{x}$ & $\mathrm{x}$ & & $\mathrm{x}$ & $\mathrm{x}$ & & & \\
\hline 347 & Furnariidae & Xenops minutus & $250-1500$ & & & $\mathrm{x}$ & $\mathrm{x}$ & & & & \\
\hline 348 & Furnariidae & Xenops rutilans & $500-1600$ & & & $\mathrm{x}$ & $\mathrm{x}$ & $\mathrm{x}$ & & $\mathrm{x}$ & \\
\hline 349 & Furnariidae & Xiphocolaptes orenocensis & $500-1100$ & & & $\mathrm{x}$ & $\mathrm{x}$ & & & & \\
\hline 350 & Furnariidae & \begin{tabular}{|l|}
$\begin{array}{l}\text { Xiphocolaptes } \\
\text { promeropirhynchus }\end{array}$ \\
\end{tabular} & $1200-2500$ & & $\mathrm{x}$ & $\mathrm{x}$ & $\mathrm{X}$ & $\mathrm{x}$ & & & \\
\hline 351 & Furnariidae & Xiphorhynchus ocellatus & $250-1350$ & & & $\mathrm{x}$ & $\mathrm{x}$ & & & & \\
\hline 352 & Furnariidae & Xiphorynchus triangularis & $1100-2400$ & & $\mathrm{x}$ & $\mathrm{x}$ & $\mathrm{x}$ & & & & $\mathrm{x}$ \\
\hline 353 & Pipridae & Chiroxiphia boliviana & $950-2000$ & & $\mathrm{x}$ & $\mathrm{x}$ & $\mathrm{x}$ & & & & $\mathrm{x}$ \\
\hline 354 & Pipridae & Chiroxiphia pareola & $<750$ & & & $\mathrm{x}$ & & & & & $\mathrm{x}$ \\
\hline 355 & Pipridae & Lepidothrix coeruleocapilla & $800-1600$ & & $\mathrm{x}$ & $\mathrm{x}$ & $\mathrm{x}$ & & & & $\mathrm{x}$ \\
\hline 356 & Pipridae & Pipra chloromeros & $250-1400$ & & & $\mathrm{x}$ & $\mathrm{x}$ & & & & $\mathrm{x}$ \\
\hline 357 & Pipridae & Piprites chloris & $250-1050$ & & & $\mathrm{x}$ & $\mathrm{x}$ & & & & \\
\hline 358 & Pipridae & Xenopipo holochlora & $450-1100$ & & & $\mathrm{x}$ & $\mathrm{x}$ & & & & \\
\hline 359 & Pipridae & Xenopipo unicolor & $1000-1800$ & & $\mathrm{x}$ & $\mathrm{x}$ & $\mathrm{x}$ & & & & \\
\hline 360 & Tyrannidae & Agriornis montana & $3350-3500$ & $\mathrm{x}$ & & & $\mathrm{x}$ & $\mathrm{x}$ & & & $\mathrm{x}$ \\
\hline 361 & Tyrannidae & Anairetes flavirostris & $1800-3600$ & $\mathrm{x}$ & $\mathrm{x}$ & & $\mathrm{x}$ & & $\mathrm{x}$ & $\mathrm{x}$ & \\
\hline 362 & Tyrannidae & Anairetes parulus & $1800-3700$ & $\mathrm{x}$ & $\mathrm{x}$ & & $\mathrm{x}$ & $\mathrm{x}$ & $\mathrm{x}$ & & \\
\hline 363 & Tyrannidae & Attila spadiceus & $250-1250$ & & & $\mathrm{x}$ & $\mathrm{x}$ & & & & \\
\hline 364 & Tyrannidae & Capsiempis flaveola & $250-1050$ & & & $\mathrm{x}$ & $\mathrm{x}$ & & & & \\
\hline
\end{tabular}




\begin{tabular}{|c|c|c|c|c|c|c|c|c|c|c|c|}
\hline 365 & Tyrannidae & Casiornis rufa & $<2000$ & & $\mathrm{x}$ & $\mathrm{x}$ & & $\mathrm{x}$ & & & \\
\hline 366 & Tyrannidae & Cnemarchus erythropygius & $3000-4300$ & $\mathrm{x}$ & & & $\mathrm{x}$ & $\mathrm{x}$ & & & \\
\hline 367 & Tyrannidae & Colonia colonus & $300-1300$ & & & $\mathrm{x}$ & $\mathrm{x}$ & & & & $\mathrm{x}$ \\
\hline 368 & Tyrannidae & Conopias cinchoneti & $900-1950$ & & $\mathrm{x}$ & $\mathrm{x}$ & $\mathrm{x}$ & $\mathrm{x}$ & & & \\
\hline 369 & Tyrannidae & Contopus cooperi & $250-2000$ & & $\mathrm{x}$ & $\mathrm{x}$ & $\mathrm{x}$ & $\mathrm{x}$ & & & \\
\hline 370 & Tyrannidae & Contopus fumigatus & $1000-2800$ & & $\mathrm{x}$ & $\mathrm{x}$ & $\mathrm{x}$ & $\mathrm{x}$ & & $\mathrm{x}$ & \\
\hline 371 & Tyrannidae & Contopus soridulus & $600-1400$ & & & $\mathrm{x}$ & $\mathrm{x}$ & $\mathrm{x}$ & & & \\
\hline 372 & Tyrannidae & Contopus virens & $250-1400$ & & & $\mathrm{x}$ & $\mathrm{x}$ & & & & \\
\hline 373 & Tyrannidae & Elaenia albiceps & $500-3250$ & $\mathrm{x}$ & $\mathrm{x}$ & $\mathrm{x}$ & $\mathrm{x}$ & & $\mathrm{x}$ & & $\mathrm{x}$ \\
\hline 374 & Tyrannidae & Elaenia chiriquensis & 3000 & & & & & & & & $\mathrm{x}$ \\
\hline 375 & Tyrannidae & Elaenia flavogaster & $250-2000$ & & $\mathrm{x}$ & $\mathrm{x}$ & $\mathrm{x}$ & & & & $\mathrm{x}$ \\
\hline 376 & Tyrannidae & Elaenia gigas & $250-1550$ & & & $\mathrm{x}$ & $\mathrm{x}$ & & & & \\
\hline 377 & Tyrannidae & Elaenia obscura & $1700-3000$ & $\mathrm{x}$ & $\mathrm{x}$ & & $\mathrm{x}$ & $\mathrm{x}$ & $\mathrm{x}$ & & \\
\hline 378 & Tyrannidae & Elaenia pallatangae & $1100-3250$ & $\mathrm{x}$ & $\mathrm{x}$ & & $\mathrm{x}$ & $\mathrm{x}$ & $\mathrm{x}$ & $\mathrm{x}$ & $\mathrm{X}$ \\
\hline 379 & Tyrannidae & Elaenia parvirostris & $250-1300$ & & & $\mathrm{x}$ & $\mathrm{x}$ & & & & \\
\hline 380 & Tyrannidae & Elaenia spectabilis & $250-1450$ & & & $\mathrm{x}$ & $\mathrm{x}$ & & & & \\
\hline 381 & Tyrannidae & Empidonax alnorum & $250-1600$ & & & $\mathrm{x}$ & $\mathrm{x}$ & $\mathrm{x}$ & & & \\
\hline 382 & Tyrannidae & $\begin{array}{l}\text { Empidonomus } \\
\text { aurantioatrocristatus }\end{array}$ & $250-1000$ & & & $\mathrm{x}$ & $\mathrm{x}$ & $\mathrm{x}$ & & & \\
\hline 383 & Tyrannidae & Hemitriccus flammulatus & $250-850$ & & & $\mathrm{x}$ & $\mathrm{x}$ & & & & \\
\hline 384 & Tyrannidae & Hemitriccus granadensis & $2000-3100$ & $\mathrm{x}$ & $\mathrm{x}$ & & $\mathrm{x}$ & $\mathrm{x}$ & & & \\
\hline 385 & Tyrannidae & Hemitriccus iohannis & $250-800$ & & & $\mathrm{x}$ & $\mathrm{x}$ & & & & \\
\hline 386 & Tyrannidae & Hemitriccus rufigularis & $750-1100$ & & & $\mathrm{x}$ & $\mathrm{x}$ & & & & \\
\hline 387 & Tyrannidae & Hirundinea ferruginea & $500-1200$ & & & $\mathrm{x}$ & $\mathrm{x}$ & $\mathrm{x}$ & & & \\
\hline 388 & Tyrannidae & Knipolegus aterrimus & $1250-3500$ & $\mathrm{x}$ & $\mathrm{x}$ & & $\mathrm{x}$ & $\mathrm{x}$ & $\mathrm{x}$ & $\mathrm{x}$ & \\
\hline 389 & Tyrannidae & Knipolegus cabanisi & $1800-2700$ & $\mathrm{x}$ & $\mathrm{x}$ & & & $\mathrm{x}$ & & & \\
\hline 390 & Tyrannidae & Knipolegus poecilurus & $900-2200$ & & $\mathrm{x}$ & $\mathrm{x}$ & $\mathrm{x}$ & $\mathrm{x}$ & & & \\
\hline
\end{tabular}




\begin{tabular}{|c|c|c|c|c|c|c|c|c|c|c|c|}
\hline 391 & Tyrannidae & Knipolegus signatus & $1000-2700$ & & $\mathrm{x}$ & $\mathrm{x}$ & $\mathrm{x}$ & & & & \\
\hline 392 & Tyrannidae & Lathrotriccus euleri & $250-2470$ & & $\mathrm{x}$ & $\mathrm{x}$ & $\mathrm{x}$ & & & $\mathrm{x}$ & \\
\hline 393 & Tyrannidae & Legatus leucophaius & $250-1200$ & & & $\mathrm{x}$ & $\mathrm{x}$ & $\mathrm{x}$ & & & \\
\hline 394 & Tyrannidae & Leptopogon amaurocephalus & $250-1100$ & & & $\mathrm{x}$ & $\mathrm{x}$ & & & & \\
\hline 395 & Tyrannidae & Leptopogon superciliaris & $600-1800$ & & $\mathrm{x}$ & $\mathrm{x}$ & $\mathrm{x}$ & $\mathrm{x}$ & & & \\
\hline 396 & Tyrannidae & Leptopogon taczanowskii & $1800-2800$ & $\mathrm{x}$ & $\mathrm{x}$ & & $\mathrm{x}$ & $\mathrm{x}$ & & & \\
\hline 397 & Tyrannidae & Lophotriccus pileatus & $800-1800$ & & $\mathrm{x}$ & $\mathrm{x}$ & $\mathrm{x}$ & $\mathrm{x}$ & & & \\
\hline 398 & Tyrannidae & Mecocerculus hellmayri & 2200 & & $\mathrm{x}$ & & $\mathrm{x}$ & & & & \\
\hline 399 & Tyrannidae & Mecocerculus leucophrys & $1800-3500$ & $\mathrm{x}$ & $\mathrm{x}$ & & $\mathrm{x}$ & $\mathrm{x}$ & & & $\mathrm{x}$ \\
\hline 400 & Tyrannidae & Mecocerculus poecilocercus & $1800-2500$ & & $\mathrm{x}$ & & & $\mathrm{x}$ & & & \\
\hline 401 & Tyrannidae & Mecocerculus stictopterus & $2400-3600$ & $\mathrm{x}$ & & & $\mathrm{x}$ & $\mathrm{x}$ & $\mathrm{x}$ & $\mathrm{x}$ & \\
\hline 402 & Tyrannidae & Mionectes oleagineus & $250-1350$ & & & $\mathrm{x}$ & $\mathrm{x}$ & & & & $\mathrm{x}$ \\
\hline 403 & Tyrannidae & Mionectes olivaceus & $250-1400$ & & & $\mathrm{x}$ & $\mathrm{x}$ & & $\mathrm{x}$ & & \\
\hline 404 & Tyrannidae & Mionectes striaticollis & $550-2600$ & & $\mathrm{x}$ & $\mathrm{x}$ & $\mathrm{x}$ & $\mathrm{x}$ & $\mathrm{x}$ & $\mathrm{x}$ & $\mathrm{x}$ \\
\hline 405 & Tyrannidae & Mitrephanes olivaceus & $1550-2100$ & & $\mathrm{x}$ & & $\mathrm{x}$ & $\mathrm{x}$ & $\mathrm{x}$ & & \\
\hline 406 & Tyrannidae & Muscisaxicola fluviatilis & $250-1600$ & & & $\mathrm{x}$ & $\mathrm{x}$ & & & & \\
\hline 407 & Tyrannidae & Muscisaxicola maculirostris & $2000-4000$ & $\mathrm{x}$ & $\mathrm{x}$ & & & $\mathrm{x}$ & & & \\
\hline 408 & Tyrannidae & Myiarchus cephalotes & $1200-1900$ & & $\mathrm{x}$ & & $\mathrm{x}$ & $\mathrm{x}$ & & $\mathrm{x}$ & \\
\hline 409 & Tyrannidae & Myiarchus ferox & $250-1100$ & & & $\mathrm{x}$ & $\mathrm{x}$ & & & $\mathrm{x}$ & \\
\hline 410 & Tyrannidae & Myiarchus tuberculifer & $250-3250$ & $\mathrm{x}$ & $\mathrm{x}$ & $\mathrm{x}$ & $\mathrm{x}$ & $\mathrm{x}$ & $\mathrm{x}$ & & \\
\hline 411 & Tyrannidae & Myiobius villosus & $650-1350$ & & & $\mathrm{x}$ & $\mathrm{x}$ & & & & $\mathrm{x}$ \\
\hline 412 & Tyrannidae & Myiodynastes chrysocephalus & $600-2470$ & & $\mathrm{x}$ & $\mathrm{x}$ & & $\mathrm{x}$ & & $\mathrm{x}$ & $\mathrm{x}$ \\
\hline 413 & Tyrannidae & Myiodynastes luteiventris & $250-1250$ & & & $\mathrm{x}$ & $\mathrm{x}$ & & & & \\
\hline 414 & Tyrannidae & Myiodynastes maculatus & $250-1500$ & & & $\mathrm{x}$ & $\mathrm{x}$ & $\mathrm{x}$ & & & $\mathrm{x}$ \\
\hline 415 & Tyrannidae & Myiopagis gaimardii & $250-900$ & & & $\mathrm{x}$ & $\mathrm{x}$ & & & & $\mathrm{x}$ \\
\hline 416 & Tyrannidae & Myiophobus fasciatus & $250-1450$ & & & $\mathrm{x}$ & $\mathrm{x}$ & & & & \\
\hline 417 & Tyrannidae & Myiophobus inornatus & $1000-2150$ & & $\mathrm{x}$ & $\mathrm{x}$ & $\mathrm{x}$ & & & & \\
\hline
\end{tabular}




\begin{tabular}{|c|c|c|c|c|c|c|c|c|c|c|c|}
\hline 418 & Tyrannidae & Myiophobus ochraceiventris & $2200-3400$ & $\mathrm{x}$ & $\mathrm{x}$ & & $\mathrm{x}$ & & & & $\mathrm{x}$ \\
\hline 419 & Tyrannidae & Myiophobus pulcher & $1500-2600$ & & $\mathrm{x}$ & & $\mathrm{x}$ & & & & \\
\hline 420 & Tyrannidae & Myiotheretes fumigatus & $2000-3400$ & $\mathrm{x}$ & $\mathrm{x}$ & & $\mathrm{x}$ & $\mathrm{x}$ & & & \\
\hline 421 & Tyrannidae & Myiotheretes fuscorufus & $2000-3400$ & $\mathrm{x}$ & $\mathrm{x}$ & & $\mathrm{x}$ & $\mathrm{x}$ & $\mathrm{x}$ & & $\mathrm{x}$ \\
\hline 422 & Tyrannidae & Myiotheretes striaticollis & $300-3500$ & $\mathrm{x}$ & $\mathrm{x}$ & $\mathrm{x}$ & $\mathrm{x}$ & & & & \\
\hline 423 & Tyrannidae & Myiotriccus ornatus & $500-1500$ & & & $\mathrm{x}$ & $\mathrm{x}$ & & & & \\
\hline 424 & Tyrannidae & Myiozetetes cayanensis & $<1000$ & & & $\mathrm{x}$ & & & & & $\mathrm{x}$ \\
\hline 425 & Tyrannidae & Myiozetetes granadensis & $250-1300$ & & & $\mathrm{x}$ & $\mathrm{x}$ & & & & $\mathrm{x}$ \\
\hline 426 & Tyrannidae & Myiozetetes similis & $250-2470$ & & $\mathrm{x}$ & $\mathrm{x}$ & $\mathrm{x}$ & $\mathrm{x}$ & & $\mathrm{x}$ & $\mathrm{x}$ \\
\hline 427 & Tyrannidae & Neopipo cinnamomea & $1500-2470$ & & $\mathrm{x}$ & & & & & $\mathrm{x}$ & \\
\hline 428 & Tyrannidae & Nephelomyias ochraceiventris & $2200-3700$ & $\mathrm{x}$ & $\mathrm{x}$ & & & $\mathrm{x}$ & & & \\
\hline 429 & Tyrannidae & Ochthoeca frontalis & $2800-3700$ & $\mathrm{x}$ & & & $\mathrm{x}$ & $\mathrm{x}$ & $\mathrm{x}$ & & \\
\hline 430 & Tyrannidae & Ochthoeca cinnamomeiventris & $1500-3300$ & $\mathrm{x}$ & $\mathrm{x}$ & & $\mathrm{x}$ & $\mathrm{x}$ & $\mathrm{x}$ & & \\
\hline 431 & Tyrannidae & Ochthoeca fumicolor & $2500-4200$ & $\mathrm{x}$ & & & $\mathrm{x}$ & $\mathrm{x}$ & $\mathrm{x}$ & & \\
\hline 432 & Tyrannidae & Ochthoeca leucophys & $2400-4200$ & $\mathrm{x}$ & & & $\mathrm{x}$ & & $\mathrm{x}$ & & \\
\hline 433 & Tyrannidae & Ochthoeca oenanthoides & $3200-4400$ & $\mathrm{x}$ & & & $\mathrm{x}$ & $\mathrm{x}$ & & & \\
\hline 434 & Tyrannidae & Ochthoeca pulchella & $1800-3100$ & $\mathrm{x}$ & $\mathrm{x}$ & & $\mathrm{x}$ & $\mathrm{x}$ & & & \\
\hline 435 & Tyrannidae & Ochthoeca rufipectoralis & $2300-4000$ & $\mathrm{x}$ & & & $\mathrm{x}$ & $\mathrm{x}$ & $\mathrm{x}$ & & $\mathrm{x}$ \\
\hline 436 & Tyrannidae & Ochthornis littoralis & $1500-2470$ & & $\mathrm{x}$ & & & & & $\mathrm{x}$ & $\mathrm{x}$ \\
\hline 437 & Tyrannidae & Ornithion inerme & $250-1000$ & & & $\mathrm{x}$ & $\mathrm{x}$ & & & & \\
\hline 438 & Tyrannidae & Phaeomyias murina & $250-500$ & & & $\mathrm{x}$ & $\mathrm{x}$ & & & & \\
\hline 439 & Tyrannidae & Phyllomyias burmeisteri & $750-1600$ & & & $\mathrm{x}$ & $\mathrm{x}$ & & & & \\
\hline 440 & Tyrannidae & Phyllomyias cinereiceps & $1300-2700$ & & $\mathrm{x}$ & $\mathrm{x}$ & $\mathrm{x}$ & $\mathrm{x}$ & & & \\
\hline 441 & Tyrannidae & Phyllomyias nigrocapillus & $1800-2100$ & & $\mathrm{x}$ & & & $\mathrm{x}$ & & & \\
\hline 442 & Tyrannidae & Phyllomyias plumbeiceps & $<2200$ & & $\mathrm{x}$ & $\mathrm{x}$ & & $\mathrm{x}$ & & & \\
\hline 443 & Tyrannidae & Phyllomyias sclateri & $1900-2200$ & & $\mathrm{x}$ & & $\mathrm{x}$ & $\mathrm{x}$ & & $\mathrm{x}$ & \\
\hline 444 & Tyrannidae & Phyllomyias uropygialis & $1800-3300$ & $\mathrm{x}$ & $\mathrm{x}$ & & $\mathrm{x}$ & $\mathrm{x}$ & & & \\
\hline
\end{tabular}




\begin{tabular}{|c|c|c|c|c|c|c|c|c|c|c|c|}
\hline 445 & Tyrannidae & Phylloscartes ophthalmicus & $750-1800$ & & $\mathrm{x}$ & $\mathrm{x}$ & $\mathrm{x}$ & $\mathrm{x}$ & & & \\
\hline 446 & Tyrannidae & Phylloscartes parkeri & $650-1550$ & & & $\mathrm{x}$ & $\mathrm{x}$ & & & & \\
\hline 447 & Tyrannidae & Phylloscartes poecilotis & $1500-2300$ & & $\mathrm{x}$ & & $\mathrm{x}$ & $\mathrm{x}$ & & & \\
\hline 448 & Tyrannidae & Phylloscartes ventralis & $1000-2400$ & & $\mathrm{x}$ & $\mathrm{x}$ & $\mathrm{x}$ & $\mathrm{x}$ & & $\mathrm{x}$ & \\
\hline 449 & Tyrannidae & Platyrinchus mystaceus & $800-1800$ & & $\mathrm{x}$ & $\mathrm{x}$ & $\mathrm{x}$ & & & & \\
\hline 450 & Tyrannidae & Poecilotriccus albifacies & $250-1050$ & & & $\mathrm{x}$ & $\mathrm{x}$ & & & & \\
\hline 451 & Tyrannidae & Poecilotriccus plumbeiceps & $1600-2150$ & & $\mathrm{x}$ & & $\mathrm{x}$ & & & & $\mathrm{x}$ \\
\hline 452 & Tyrannidae & Poecilotriccus pulchellus & $600-1500$ & & & $\mathrm{x}$ & $\mathrm{x}$ & & & & $\mathrm{x}$ \\
\hline 453 & Tyrannidae & Pseudotriccus ruficeps & $2000-3350$ & $\mathrm{x}$ & $\mathrm{x}$ & & $\mathrm{x}$ & $\mathrm{x}$ & & & \\
\hline 454 & Tyrannidae & Pseudotriccus simplex & 1100-3055 & $\mathrm{x}$ & $\mathrm{x}$ & $\mathrm{x}$ & $\mathrm{x}$ & & & & \\
\hline 455 & Tyrannidae & Pyrocephalus rubinus & $<1000$ & & & $\mathrm{x}$ & & & & & $\mathrm{x}$ \\
\hline 456 & Tyrannidae & Pyrrhomyias cinnamomea & $900-3400$ & $\mathrm{x}$ & $\mathrm{x}$ & $\mathrm{x}$ & $\mathrm{x}$ & $\mathrm{x}$ & $\mathrm{x}$ & & $\mathrm{x}$ \\
\hline 457 & Tyrannidae & Ramphotrigon megacephala & $250-1200$ & & & $\mathrm{x}$ & $\mathrm{x}$ & & & & \\
\hline 458 & Tyrannidae & Rhynchocyclus fulvipectus & $1000-2000$ & & $\mathrm{x}$ & $\mathrm{x}$ & $\mathrm{x}$ & & & & \\
\hline 459 & Tyrannidae & Rhynchocyclus olivaceus & $250-1000$ & & & $\mathrm{x}$ & $\mathrm{x}$ & & & & \\
\hline 460 & Tyrannidae & Rhytipterna simplex & $250-1400$ & & & $\mathrm{x}$ & $\mathrm{x}$ & & & & \\
\hline 461 & Tyrannidae & Sayornis nigricans & $600-3000$ & $\mathrm{x}$ & $\mathrm{x}$ & $\mathrm{x}$ & $\mathrm{x}$ & & $\mathrm{x}$ & $\mathrm{x}$ & \\
\hline 462 & Tyrannidae & Serpophaga cinerea & $900-3100$ & $\mathrm{x}$ & $\mathrm{x}$ & $\mathrm{x}$ & $\mathrm{x}$ & & $\mathrm{x}$ & $\mathrm{x}$ & $\mathrm{x}$ \\
\hline 463 & Thraupidae & Tachyphonus rufus & 2600- 3055 & $\mathrm{x}$ & & & & & & & $\mathrm{x}$ \\
\hline 464 & Tyrannidae & Tolmomyias flaviventris & $250-1500$ & & & $\mathrm{x}$ & $\mathrm{x}$ & & & & $\mathrm{x}$ \\
\hline 465 & Tyrannidae & Tolmomyias poliocephalus & $250-3055$ & $\mathrm{x}$ & $\mathrm{x}$ & $\mathrm{x}$ & $\mathrm{x}$ & & & $\mathrm{x}$ & \\
\hline 466 & Tyrannidae & Tolmomyias sulphurescens & $2000-2300$ & & $\mathrm{x}$ & & $\mathrm{x}$ & $\mathrm{x}$ & & & \\
\hline 467 & Tyrannidae & Tyranneutes stolzmanni & & & & & & & & & $\mathrm{x}$ \\
\hline 468 & Tyrannidae & Tyrannus melancholicus & $250-2600$ & & $\mathrm{x}$ & $\mathrm{x}$ & $\mathrm{x}$ & & & $\mathrm{x}$ & $\mathrm{x}$ \\
\hline 469 & Tyrannidae & Uromyias agraphia & $2700-3400$ & $\mathrm{x}$ & & & & $\mathrm{x}$ & & & \\
\hline 470 & Tyrannidae & Zimmerius bolivianus & $1000-2600$ & & $\mathrm{x}$ & $\mathrm{x}$ & $\mathrm{x}$ & $\mathrm{x}$ & & $\mathrm{x}$ & $\mathrm{X}$ \\
\hline 471 & Tyrannidae & Zimmerius cinereicapillus & $550-1300$ & & & $\mathrm{x}$ & $\mathrm{x}$ & & & & \\
\hline
\end{tabular}




\begin{tabular}{|c|c|c|c|c|c|c|c|c|c|c|c|}
\hline 472 & Cotingidae & Ampelioides tschudii & $700-1400$ & & & $\mathrm{x}$ & $\mathrm{x}$ & & & & \\
\hline 473 & Cotingidae & Ampelion rubrocristata & $2400-3700$ & $\mathrm{x}$ & & & $\mathrm{x}$ & $\mathrm{x}$ & $\mathrm{x}$ & & $\mathrm{x}$ \\
\hline 474 & Cotingidae & Ampelion rufaxilla & $1800-2700$ & $\mathrm{x}$ & $\mathrm{x}$ & & $\mathrm{x}$ & $\mathrm{x}$ & & $\mathrm{x}$ & \\
\hline 475 & Cotingidae & Cephalopterus ornatus & $250-1650$ & & & $\mathrm{x}$ & $\mathrm{x}$ & & & & $\mathrm{x}$ \\
\hline 476 & Cotingidae & Cotinga maynana & $250-1000$ & & & $\mathrm{x}$ & $\mathrm{x}$ & & & & \\
\hline 477 & Cotingidae & Oxyruncus cristatus & $850-1300$ & & & $\mathrm{x}$ & $\mathrm{x}$ & & & & \\
\hline 478 & Cotingidae & Pipreola arcuata & $2100-3500$ & $\mathrm{x}$ & $\mathrm{x}$ & & $\mathrm{x}$ & $\mathrm{x}$ & & & $\mathrm{x}$ \\
\hline 479 & Cotingidae & Pipreola frontalis & $1000-1500$ & & & $\mathrm{x}$ & $\mathrm{x}$ & & & & \\
\hline 480 & Cotingidae & Pipreola intermedia & $2000-3000$ & $\mathrm{x}$ & $\mathrm{x}$ & & $\mathrm{x}$ & $\mathrm{x}$ & & & \\
\hline 481 & Cotingidae & Pipreola pulchra & $1800-2400$ & & $\mathrm{x}$ & & & $\mathrm{x}$ & & & \\
\hline 482 & Cotingidae & Rupicola peruviana & $900-2400$ & & $\mathrm{x}$ & $\mathrm{x}$ & $\mathrm{x}$ & $\mathrm{x}$ & & $\mathrm{x}$ & $\mathrm{x}$ \\
\hline 483 & Cotingidae & Snowornis subalaris & $850-1350$ & & & $\mathrm{x}$ & $\mathrm{x}$ & & & & \\
\hline 484 & Tityridae & Laniisoma elegans & $750-1400$ & & & $\mathrm{x}$ & $\mathrm{x}$ & & & & \\
\hline 485 & Tityridae & Pachyramphus albogriseus & 1500 & & & $\mathrm{x}$ & $\mathrm{x}$ & & & & \\
\hline 486 & Tityridae & Pachyramphus polychopterus & $250-1100$ & & & $\mathrm{x}$ & $\mathrm{x}$ & & & $\mathrm{x}$ & \\
\hline 487 & Tityridae & Pachyramphus validus & $<2000$ & & $\mathrm{x}$ & $\mathrm{x}$ & & $\mathrm{x}$ & & & \\
\hline 488 & Tityridae & Pachyramphus versicolor & $1800-3000$ & $\mathrm{x}$ & $\mathrm{x}$ & & $\mathrm{x}$ & $\mathrm{x}$ & & $\mathrm{x}$ & \\
\hline 489 & Tityridae & Pachyramphus viridis & 900 & & & $\mathrm{x}$ & $\mathrm{x}$ & & & & \\
\hline 490 & Tityridae & Schiffornis turdinus & $250-1400$ & & & $\mathrm{x}$ & $\mathrm{x}$ & & & & \\
\hline 491 & Tityridae & Tityra semifasciata & $250-1500$ & & & $\mathrm{x}$ & $\mathrm{x}$ & & & & $\mathrm{x}$ \\
\hline 492 & Vireonidae & Hylophilus hypoxanthus & $1500-2470$ & & $\mathrm{x}$ & & $\mathrm{x}$ & & & $\mathrm{x}$ & \\
\hline 493 & Vireonidae & Hylophilus ochraceiceps & $250-1050$ & & & $\mathrm{x}$ & $\mathrm{x}$ & & & & \\
\hline 494 & Vireonidae & Vireo leucophrys & $1800-2600$ & & $\mathrm{x}$ & & $\mathrm{x}$ & $\mathrm{x}$ & & $\mathrm{x}$ & \\
\hline 495 & Vireonidae & Vireo olivaceus & $<2600$ & & $\mathrm{x}$ & $\mathrm{x}$ & $\mathrm{x}$ & $\mathrm{x}$ & $\mathrm{x}$ & $\mathrm{x}$ & $\mathrm{x}$ \\
\hline 496 & Vireonidae & Vireolanius leucotis & $250-1500$ & & & $\mathrm{x}$ & $\mathrm{x}$ & & & & \\
\hline 497 & Corvidae & Cyanolyca viridicyana & $2200-3500$ & $\mathrm{x}$ & $\mathrm{x}$ & & $\mathrm{x}$ & $\mathrm{x}$ & & & $\mathrm{x}$ \\
\hline 498 & Corvidae & Cyanocorax violaceus & $250-1400$ & & & $\mathrm{x}$ & $\mathrm{x}$ & & & $\mathrm{x}$ & $\mathrm{x}$ \\
\hline
\end{tabular}




\begin{tabular}{|c|c|c|c|c|c|c|c|c|c|c|c|}
\hline 499 & Corvidae & Cyanocorax yncas & $<2400$ & & $\mathrm{x}$ & $\mathrm{x}$ & $\mathrm{x}$ & $\mathrm{x}$ & & $\mathrm{x}$ & \\
\hline 500 & Hirundinidae & Hapalochelidon andecola & $3450-3500$ & $\mathrm{x}$ & & & $\mathrm{x}$ & & & & \\
\hline 501 & Hirundinidae & Hirundo rustica & $900-4000$ & $\mathrm{x}$ & $\mathrm{x}$ & $\mathrm{x}$ & $\mathrm{x}$ & & $\mathrm{x}$ & & \\
\hline 502 & Hirundinidae & Neochelidon tibialis & $250-1250$ & & & $\mathrm{x}$ & $\mathrm{x}$ & & & & \\
\hline 503 & Hirundinidae & Orochelidon flavipes & $2000-3500$ & $\mathrm{x}$ & $\mathrm{x}$ & & $\mathrm{x}$ & $\mathrm{x}$ & & & \\
\hline 504 & Hirundinidae & Orochelidon murina & $2500-4600$ & $\mathrm{x}$ & & & $\mathrm{x}$ & $\mathrm{x}$ & & & \\
\hline 505 & Hirundinidae & Petrochelidon pyrrhonota & $2000-3300$ & $\mathrm{x}$ & $\mathrm{x}$ & & & $\mathrm{x}$ & & & \\
\hline 506 & Hirundinidae & Progne tapera & $250-1400$ & & & $\mathrm{x}$ & $\mathrm{x}$ & & & & \\
\hline 507 & Hirundinidae & Pygochelidon cyanoleuca & $900-4300$ & $\mathrm{x}$ & $\mathrm{x}$ & $\mathrm{x}$ & $\mathrm{x}$ & $\mathrm{x}$ & $\mathrm{x}$ & & $\mathrm{x}$ \\
\hline 508 & Hirundinidae & Stelgidopteryx ruficollis & $250-1600$ & & & $\mathrm{x}$ & $\mathrm{x}$ & & & & $\mathrm{x}$ \\
\hline 509 & Troglodytidae & Cinnycerthia fulva & $1800-3000$ & $\mathrm{x}$ & $\mathrm{x}$ & & $\mathrm{x}$ & $\mathrm{x}$ & $\mathrm{x}$ & & \\
\hline 510 & Troglodytidae & $\begin{array}{l}\text { Cistothorus (minimus) } \\
\text { platensis }\end{array}$ & $3500-4600$ & $\mathrm{x}$ & & & $\mathrm{x}$ & $\mathrm{x}$ & & & \\
\hline 511 & Troglodytidae & Cyphorhinus thoracicus & $800-1600$ & & & $\mathrm{x}$ & $\mathrm{x}$ & & & & \\
\hline 512 & Troglodytidae & Henicorhina leucophrys & $1800-2800$ & $\mathrm{x}$ & $\mathrm{x}$ & & $\mathrm{x}$ & $\mathrm{x}$ & & & $\mathrm{x}$ \\
\hline 513 & Troglodytidae & Microcerculus marginatus & $250-1250$ & & & $\mathrm{x}$ & $\mathrm{x}$ & & & & \\
\hline 514 & Troglodytidae & Odontorchilus branickii & $<2000$ & & $\mathrm{x}$ & $\mathrm{x}$ & $\mathrm{x}$ & $\mathrm{x}$ & & & \\
\hline 515 & Troglodytidae & Pheugopedius eisenmanni & $1800-3400$ & $\mathrm{x}$ & $\mathrm{x}$ & & & $\mathrm{x}$ & & $\mathrm{x}$ & \\
\hline 516 & Troglodytidae & Thryothorus genibarbis & $250-1500$ & & & $\mathrm{x}$ & $\mathrm{x}$ & & & & \\
\hline 517 & Troglodytidae & Troglodytes aedon & $1500-3055$ & $\mathrm{x}$ & $\mathrm{x}$ & & $\mathrm{x}$ & & $\mathrm{x}$ & $\mathrm{x}$ & $\mathrm{x}$ \\
\hline 518 & Troglodytidae & Troglodytes solstitialis & $2000-3600$ & $\mathrm{x}$ & $\mathrm{x}$ & & $\mathrm{x}$ & $\mathrm{x}$ & $\mathrm{x}$ & & $\mathrm{x}$ \\
\hline 519 & Polioptilidae & Microbates cinereiventris & $450-1000$ & & & $\mathrm{x}$ & $\mathrm{x}$ & & & & \\
\hline 520 & Cinclidae & Cinclus leucocephalus & $900-4200$ & $\mathrm{x}$ & $\mathrm{x}$ & $\mathrm{x}$ & $\mathrm{x}$ & $\mathrm{x}$ & $\mathrm{x}$ & $\mathrm{x}$ & $\mathrm{x}$ \\
\hline 521 & Turdidae & Catharus dryas & $700-1500$ & & & $\mathrm{x}$ & $\mathrm{x}$ & & & & $\mathrm{x}$ \\
\hline 522 & Turdidae & Catharus fuscater & $1800-2900$ & & $\mathrm{x}$ & & $\mathrm{x}$ & $\mathrm{x}$ & & & \\
\hline 523 & Turdidae & Catharus ustulatus & $<3500$ & $\mathrm{x}$ & $\mathrm{x}$ & & $\mathrm{x}$ & $\mathrm{x}$ & & & \\
\hline 524 & Turdidae & Entomodestes leucotis & $1800-2900$ & $\mathrm{x}$ & $\mathrm{x}$ & & $\mathrm{x}$ & $\mathrm{x}$ & & & $\mathrm{x}$ \\
\hline
\end{tabular}




\begin{tabular}{|c|c|c|c|c|c|c|c|c|c|c|c|}
\hline 525 & Turdidae & Myadestes ralloides & $1800-2800$ & $\mathrm{x}$ & $\mathrm{x}$ & & $\mathrm{x}$ & $\mathrm{x}$ & & & \\
\hline 526 & Turdidae & Turdus albicollis & $250-850$ & & & $\mathrm{x}$ & $\mathrm{x}$ & & & & \\
\hline 527 & Turdidae & Turdus chiguanco & $1300-4300$ & $\mathrm{x}$ & $\mathrm{x}$ & & $\mathrm{x}$ & & $\mathrm{x}$ & $\mathrm{x}$ & $\mathrm{x}$ \\
\hline 528 & Turdidae & Turdus fuscater & $2500-4200$ & $\mathrm{x}$ & & & $\mathrm{x}$ & $\mathrm{x}$ & $\mathrm{x}$ & $\mathrm{x}$ & $\mathrm{X}$ \\
\hline 529 & Turdidae & Turdus ignobilis & $<2000$ & & $\mathrm{x}$ & & $\mathrm{x}$ & $\mathrm{x}$ & & & \\
\hline 530 & Turdidae & Turdus leucops & $1800-2000$ & & $\mathrm{x}$ & & $\mathrm{x}$ & $\mathrm{x}$ & & & \\
\hline 531 & Turdidae & Turdus nigriceps & $<2000$ & & $\mathrm{x}$ & & $\mathrm{x}$ & $\mathrm{x}$ & $\mathrm{x}$ & & $\mathrm{x}$ \\
\hline 532 & Turdidae & Turdus serranus & $1800-3000$ & $\mathrm{x}$ & $\mathrm{x}$ & & $\mathrm{x}$ & $\mathrm{x}$ & $\mathrm{x}$ & $\mathrm{x}$ & $\mathrm{x}$ \\
\hline 533 & Motacillidae & Anthus bogotensis & $3450-3500$ & $\mathrm{x}$ & & & $\mathrm{x}$ & & & & \\
\hline 534 & Thraupidae & Anisognathus igniventris & $2400-3600$ & $\mathrm{x}$ & & & $\mathrm{x}$ & $\mathrm{x}$ & $\mathrm{x}$ & $\mathrm{x}$ & $\mathrm{x}$ \\
\hline 535 & Thraupidae & Anisognathus lacrymosus & $1800-3500$ & $\mathrm{x}$ & $\mathrm{x}$ & & & $\mathrm{x}$ & & & \\
\hline 536 & Thraupidae & Anisognathus somptuosus & $1500-2470$ & & $\mathrm{x}$ & & $\mathrm{x}$ & & & $\mathrm{x}$ & \\
\hline 537 & Thraupidae & Buthraupis montana & $2300-3500$ & $\mathrm{x}$ & $\mathrm{x}$ & & $\mathrm{x}$ & $\mathrm{x}$ & $\mathrm{x}$ & $\mathrm{x}$ & $\mathrm{x}$ \\
\hline 538 & Thraupidae & Catamblyrhynchus diadema & $2000-3500$ & $\mathrm{x}$ & $\mathrm{x}$ & & $\mathrm{x}$ & $\mathrm{x}$ & & & \\
\hline 539 & Thraupidae & Catamenia analis & $900-4000$ & $\mathrm{x}$ & $\mathrm{x}$ & $\mathrm{x}$ & $\mathrm{x}$ & $\mathrm{x}$ & $\mathrm{x}$ & & \\
\hline 540 & Thraupidae & Catamenia homochroa & $2350-3500$ & $\mathrm{x}$ & & & $\mathrm{x}$ & $\mathrm{x}$ & & & \\
\hline 541 & Thraupidae & Catamenia inornata & $2600-4400$ & $\mathrm{x}$ & & & $\mathrm{x}$ & $\mathrm{x}$ & $\mathrm{x}$ & & \\
\hline 542 & Thraupidae & Chlorochrysa calliparaea & $<2200$ & & $\mathrm{x}$ & & $\mathrm{x}$ & $\mathrm{x}$ & & & $\mathrm{x}$ \\
\hline 543 & Thraupidae & Chlorophanes spiza & $250-1200$ & & & $\mathrm{x}$ & $\mathrm{x}$ & & & & $\mathrm{x}$ \\
\hline 544 & Thraupidae & Chlorornis riefferii & $2000-3500$ & $\mathrm{x}$ & $\mathrm{x}$ & & $\mathrm{x}$ & $\mathrm{x}$ & & $\mathrm{x}$ & $\mathrm{x}$ \\
\hline 545 & Thraupidae & Chlorospingus flavigularis & $<1000$ & & & $\mathrm{x}$ & & & & & $\mathrm{x}$ \\
\hline 546 & Thraupidae & Cissopis leverianus & $250-1600$ & & & $\mathrm{x}$ & $\mathrm{x}$ & & & & $\mathrm{x}$ \\
\hline 547 & Thraupidae & Cnemoscopus rubrirostris & $2000-3000$ & $\mathrm{x}$ & $\mathrm{x}$ & & $\mathrm{x}$ & $\mathrm{x}$ & & $\mathrm{x}$ & \\
\hline 548 & Thraupidae & Coereba flaveola & $250-1500$ & & & $\mathrm{x}$ & $\mathrm{x}$ & & & & \\
\hline 549 & Thraupidae & Conirostrum albifrons & $1800-3000$ & $\mathrm{x}$ & $\mathrm{x}$ & & $\mathrm{x}$ & $\mathrm{x}$ & $\mathrm{x}$ & & \\
\hline 550 & Thraupidae & Conirostrum cinereum & $900-4200$ & $\mathrm{x}$ & $\mathrm{x}$ & $\mathrm{x}$ & $\mathrm{x}$ & & $\mathrm{x}$ & & \\
\hline 551 & Thraupidae & Conirostrum ferrugineiventre & $2600-4100$ & $\mathrm{x}$ & & & $\mathrm{x}$ & $\mathrm{x}$ & & & \\
\hline
\end{tabular}




\begin{tabular}{|c|c|c|c|c|c|c|c|c|c|c|c|}
\hline 552 & Thraupidae & Conirostrum sitticolor & $2300-3600$ & $\mathrm{x}$ & $\mathrm{x}$ & & $\mathrm{x}$ & & & & $\mathrm{x}$ \\
\hline 553 & Thraupidae & Corydospiza alaudina & $900-4100$ & $\mathrm{x}$ & $\mathrm{x}$ & $\mathrm{x}$ & & & $\mathrm{x}$ & & \\
\hline 554 & Thraupidae & Creurgops dentatus & $<2500$ & & $\mathrm{x}$ & & $\mathrm{x}$ & $\mathrm{x}$ & & $\mathrm{x}$ & \\
\hline 555 & Thraupidae & Cyanerpes caeruleus & $250-1400$ & & & $\mathrm{x}$ & $\mathrm{x}$ & & & & $\mathrm{x}$ \\
\hline 556 & Thraupidae & Dacnis cayana & $<2000$ & & $\mathrm{x}$ & & $\mathrm{x}$ & $\mathrm{x}$ & & & $\mathrm{x}$ \\
\hline 557 & Thraupidae & Dacnis lineata & $250-1300$ & & & $\mathrm{x}$ & $\mathrm{x}$ & & & & $\mathrm{x}$ \\
\hline 558 & Thraupidae & Delothraupis castaneoventris & $2600-3500$ & $\mathrm{x}$ & $\mathrm{x}$ & & $\mathrm{x}$ & & $\mathrm{x}$ & & \\
\hline 559 & Thraupidae & Diglossa brunneiventris & $2400-4300$ & $\mathrm{x}$ & & & $\mathrm{x}$ & $\mathrm{x}$ & $\mathrm{x}$ & $\mathrm{x}$ & $\mathrm{x}$ \\
\hline 560 & Thraupidae & Diglossa caerulescens & $1800-3100$ & $\mathrm{x}$ & $\mathrm{x}$ & & $\mathrm{x}$ & $\mathrm{x}$ & & & \\
\hline 561 & Thraupidae & Diglossa cyanea & $1800-3600$ & $\mathrm{x}$ & $\mathrm{x}$ & & $\mathrm{x}$ & $\mathrm{x}$ & $\mathrm{x}$ & & \\
\hline 562 & Thraupidae & Diglossa glauca & $1000-2300$ & & $\mathrm{x}$ & & $\mathrm{x}$ & & & & $\mathrm{x}$ \\
\hline 563 & Thraupidae & Diglossa mystacalis & $2500-3700$ & $\mathrm{x}$ & & & $\mathrm{x}$ & $\mathrm{x}$ & $\mathrm{x}$ & & \\
\hline 564 & Thraupidae & Diglossa sittoides & $1800-3500$ & $\mathrm{x}$ & $\mathrm{x}$ & & $\mathrm{x}$ & $\mathrm{x}$ & $\mathrm{x}$ & $\mathrm{x}$ & $\mathrm{x}$ \\
\hline 565 & Thraupidae & Dubusia castaneoventris & $2000-3500$ & $\mathrm{x}$ & $\mathrm{x}$ & & & $\mathrm{x}$ & $\mathrm{x}$ & & \\
\hline 566 & Thraupidae & Dubusia taeniata & $1900-3400$ & $\mathrm{x}$ & $\mathrm{x}$ & & $\mathrm{x}$ & $\mathrm{x}$ & & & \\
\hline 567 & Thraupidae & Geospizopsis plebejus & $2400-4700$ & $\mathrm{x}$ & $\mathrm{x}$ & & & $\mathrm{x}$ & $\mathrm{x}$ & & $\mathrm{x}$ \\
\hline 568 & Thraupidae & Geospizopsis unicolor & $3000-4700$ & $\mathrm{x}$ & $\mathrm{x}$ & & $\mathrm{x}$ & $\mathrm{x}$ & $\mathrm{x}$ & & $\mathrm{x}$ \\
\hline 569 & Thraupidae & Haplospiza rustica & $1800-3300$ & $\mathrm{x}$ & $\mathrm{x}$ & & $\mathrm{x}$ & $\mathrm{x}$ & & & \\
\hline 570 & Thraupidae & $\begin{array}{l}\text { Hemispingus (atropileus) } \\
\text { auricularis }\end{array}$ & $2600-3700$ & $\mathrm{x}$ & & & $\mathrm{X}$ & $\mathrm{x}$ & & & \\
\hline 571 & Thraupidae & Hemispingus frontalis & $1800-2600$ & & $\mathrm{x}$ & & $\mathrm{x}$ & $\mathrm{x}$ & $\mathrm{x}$ & & \\
\hline 572 & Thraupidae & Hemispingus melanotis & $1800-2200$ & & $\mathrm{x}$ & & $\mathrm{x}$ & $\mathrm{x}$ & $\mathrm{x}$ & & $\mathrm{x}$ \\
\hline 573 & Thraupidae & Hemispingus parodii & $2600-3500$ & $\mathrm{x}$ & & & & $\mathrm{x}$ & & & \\
\hline 574 & Thraupidae & Hemispingus superciliaris & $2200-3450$ & $\mathrm{x}$ & $\mathrm{x}$ & & $\mathrm{x}$ & $\mathrm{x}$ & & & \\
\hline 575 & Thraupidae & Hemispingus trifasciatus & $3000-3700$ & $\mathrm{x}$ & & & $\mathrm{x}$ & $\mathrm{x}$ & $\mathrm{x}$ & & \\
\hline 576 & Thraupidae & Hemispingus xanthophthalmus & $2200-3500$ & $\mathrm{x}$ & $\mathrm{x}$ & & $\mathrm{x}$ & $\mathrm{x}$ & & & \\
\hline 577 & Thraupidae & Iridophanes pulcherrimus & $1100-1800$ & & $\mathrm{x}$ & & $\mathrm{x}$ & & & & \\
\hline
\end{tabular}




\begin{tabular}{|c|c|c|c|c|c|c|c|c|c|c|c|}
\hline 578 & Thraupidae & Iridosornis analis & $1800-2300$ & & $\mathrm{x}$ & & $\mathrm{x}$ & $\mathrm{x}$ & & & $\mathrm{x}$ \\
\hline 579 & Thraupidae & Iridosornis jelskii & $3000-3700$ & $\mathrm{x}$ & & & $\mathrm{x}$ & $\mathrm{x}$ & $\mathrm{x}$ & & \\
\hline 580 & Thraupidae & Iridosornis reinhardti & $2100-3700$ & $\mathrm{x}$ & $\mathrm{x}$ & & & $\mathrm{x}$ & $\mathrm{x}$ & & \\
\hline 581 & Thraupidae & Parkerthraustes humeralis & $250-650$ & & & $\mathrm{x}$ & $\mathrm{x}$ & & & & \\
\hline 582 & Thraupidae & Pipraeidea bonariensis & $1800-4000$ & $\mathrm{x}$ & $\mathrm{x}$ & & $\mathrm{x}$ & $\mathrm{x}$ & $\mathrm{x}$ & & \\
\hline 583 & Thraupidae & Pipraeidea melanonota & $<3000$ & $\mathrm{x}$ & & & $\mathrm{x}$ & $\mathrm{x}$ & & & \\
\hline 584 & Thraupidae & Poospizopsis caesar & $3000-3800$ & $\mathrm{x}$ & & & & $\mathrm{x}$ & $\mathrm{x}$ & & $\mathrm{x}$ \\
\hline 585 & Thraupidae & Ramphocelus carbo & 1683- 3055 & $\mathrm{x}$ & $\mathrm{x}$ & & $\mathrm{x}$ & $\mathrm{x}$ & & $\mathrm{x}$ & $\mathrm{x}$ \\
\hline 586 & Thraupidae & Rhopospina fruticeti & $2300-4200$ & $\mathrm{x}$ & $\mathrm{x}$ & & $\mathrm{X}$ & & $\mathrm{x}$ & & $\mathrm{X}$ \\
\hline 587 & Thraupidae & Saltator aurantiirostris & $2100-4000$ & $\mathrm{x}$ & $\mathrm{x}$ & & $\mathrm{x}$ & $\mathrm{x}$ & $\mathrm{x}$ & & \\
\hline 588 & Thraupidae & Saltator maximus & $<2000$ & & $\mathrm{x}$ & & $\mathrm{x}$ & $\mathrm{x}$ & & $\mathrm{x}$ & $\mathrm{x}$ \\
\hline 589 & Thraupidae & Schistochlamys melanopis & $600-2600$ & & $\mathrm{x}$ & $\mathrm{x}$ & $\mathrm{x}$ & & & & \\
\hline 590 & Thraupidae & Sicalis olivascens & $1650-4200$ & $\mathrm{x}$ & $\mathrm{x}$ & & & $\mathrm{x}$ & $\mathrm{x}$ & & \\
\hline 591 & Thraupidae & Sporophila angolensis & $1500-2470$ & & $\mathrm{x}$ & & & & & $\mathrm{x}$ & $\mathrm{x}$ \\
\hline 592 & Thraupidae & Sporophila castaneiventris & $250-1450$ & & & $\mathrm{x}$ & $\mathrm{x}$ & & & & \\
\hline 593 & Thraupidae & Sporophila luctuosa & $900-3200$ & $\mathrm{x}$ & $\mathrm{x}$ & & $\mathrm{x}$ & $\mathrm{x}$ & $\mathrm{x}$ & & \\
\hline 594 & Thraupidae & Sporophila nigricollis & $900-2600$ & & $\mathrm{x}$ & $\mathrm{x}$ & $\mathrm{x}$ & $\mathrm{x}$ & $\mathrm{x}$ & $\mathrm{x}$ & \\
\hline 595 & Thraupidae & Tachyphonus rufiventer & $250-1250$ & & & $\mathrm{x}$ & $\mathrm{x}$ & & & & $\mathrm{x}$ \\
\hline 596 & Thraupidae & Tangara argyrofenges & 1300 & & & $\mathrm{x}$ & $\mathrm{x}$ & & & & \\
\hline 597 & Thraupidae & Tangara arthus & $750-1800$ & & & & $\mathrm{x}$ & & & & $\mathrm{x}$ \\
\hline 598 & Thraupidae & Tangara chilensis & $250-1600$ & & & & $\mathrm{x}$ & & & & $\mathrm{x}$ \\
\hline 599 & Thraupidae & Tangara chrysotis & $850-1600$ & & & $\mathrm{x}$ & $\mathrm{x}$ & & & & \\
\hline 600 & Thraupidae & Tangara cyanicollis & $1800-2400$ & & $\mathrm{x}$ & & $\mathrm{x}$ & $\mathrm{x}$ & & $\mathrm{x}$ & $\mathrm{x}$ \\
\hline 601 & Thraupidae & Tangara cyanotis & $1300-2000$ & & $\mathrm{x}$ & $\mathrm{x}$ & $\mathrm{x}$ & & & & \\
\hline 602 & Thraupidae & Tangara gyrola & $<2000$ & & $\mathrm{x}$ & & $\mathrm{x}$ & $\mathrm{x}$ & & $\mathrm{x}$ & $\mathrm{x}$ \\
\hline 603 & Thraupidae & Tangara nigrocincta & $250-1100$ & & & $\mathrm{x}$ & $\mathrm{x}$ & & & & \\
\hline 604 & Thraupidae & Tangara nigroviridis & $1800-2500$ & & $\mathrm{x}$ & & $\mathrm{x}$ & $\mathrm{x}$ & & $\mathrm{x}$ & $\mathrm{X}$ \\
\hline
\end{tabular}




\begin{tabular}{|c|c|c|c|c|c|c|c|c|c|c|c|}
\hline 605 & Thraupidae & Tangara parzudakii & $1800-2600$ & & $\mathrm{x}$ & & $\mathrm{x}$ & $\mathrm{x}$ & & & \\
\hline 606 & Thraupidae & Tangara punctata & $600-2000$ & & $\mathrm{x}$ & $\mathrm{x}$ & $\mathrm{x}$ & & & & $\mathrm{x}$ \\
\hline 607 & Thraupidae & Tangara ruficervix & $1800-2400$ & & $\mathrm{x}$ & & $\mathrm{x}$ & $\mathrm{x}$ & & & \\
\hline 608 & Thraupidae & Tangara vassorii & $2400-3500$ & $\mathrm{x}$ & & & $\mathrm{x}$ & $\mathrm{x}$ & $\mathrm{x}$ & & $\mathrm{x}$ \\
\hline 609 & Thraupidae & Tangara viridicollis & $1800-2700$ & & $\mathrm{x}$ & & $\mathrm{x}$ & $\mathrm{x}$ & & $\mathrm{x}$ & \\
\hline 610 & Thraupidae & Tangara xanthocephala & $1800-2400$ & & $\mathrm{x}$ & & $\mathrm{x}$ & & & $\mathrm{x}$ & $\mathrm{x}$ \\
\hline 611 & Thraupidae & Tangara xanthogastra & $250-1300$ & & $\mathrm{x}$ & & $\mathrm{x}$ & $\mathrm{x}$ & & & \\
\hline 612 & Thraupidae & Tersina viridis & $<1000$ & & $\mathrm{x}$ & & $\mathrm{x}$ & & & & $\mathrm{x}$ \\
\hline 613 & Thraupidae & Thlypopsis ornata & $1600-3800$ & $\mathrm{x}$ & $\mathrm{x}$ & & $\mathrm{x}$ & & $\mathrm{x}$ & $\mathrm{x}$ & \\
\hline 614 & Thraupidae & Thlypopsis ruficeps & $1800-3700$ & $\mathrm{x}$ & $\mathrm{x}$ & & $\mathrm{x}$ & $\mathrm{x}$ & $\mathrm{x}$ & $\mathrm{x}$ & $\mathrm{X}$ \\
\hline 615 & Thraupidae & Thraupis cyanocephala & $1800-3100$ & $\mathrm{x}$ & $\mathrm{x}$ & & $\mathrm{x}$ & $\mathrm{x}$ & $\mathrm{x}$ & $\mathrm{x}$ & $\mathrm{x}$ \\
\hline 616 & Thraupidae & Thraupis episcopus & $<2000$ & & $\mathrm{x}$ & & $\mathrm{x}$ & & & $\mathrm{x}$ & $\mathrm{x}$ \\
\hline 617 & Thraupidae & Thraupis palmarum & $250-1600$ & & $\mathrm{x}$ & $\mathrm{x}$ & $\mathrm{x}$ & & & & $\mathrm{x}$ \\
\hline 618 & Thraupidae & Trichothraupis melanops & $1000-1600$ & & & $\mathrm{x}$ & $\mathrm{x}$ & & & & \\
\hline 619 & Thraupidae & Xenodacnis parina & $3450-3500$ & $\mathrm{x}$ & & & $\mathrm{x}$ & & & & \\
\hline 620 & Emberizidae & Ammodramus aurifrons & $250-1600$ & & & $\mathrm{x}$ & $\mathrm{x}$ & & & & \\
\hline 621 & Emberizidae & Arremon assimilis & $2000-3400$ & $\mathrm{x}$ & $\mathrm{x}$ & & & $\mathrm{x}$ & & & \\
\hline 622 & Emberizidae & Arremon brunneinucha & $1500-3055$ & $\mathrm{x}$ & $\mathrm{x}$ & & $\mathrm{x}$ & $\mathrm{x}$ & & $\mathrm{x}$ & $x$ \\
\hline 623 & Emberizidae & Atlapetes canigenis & $2450-3000$ & $\mathrm{x}$ & & & & $\mathrm{x}$ & $\mathrm{x}$ & & \\
\hline 624 & Emberizidae & Atlapetes forbesi & $2700-4600$ & $\mathrm{x}$ & & & & & $\mathrm{x}$ & & \\
\hline 625 & Emberizidae & Atlapetes melanolaemus & 1750 & & $\mathrm{x}$ & & $\mathrm{x}$ & & & & $\mathrm{x}$ \\
\hline 626 & Emberizidae & Atlapetes rufinucha & 1750 & & $\mathrm{x}$ & & & & & & $\mathrm{x}$ \\
\hline 627 & Emberizidae & Atlaptes tricolor & $1800-3050$ & $\mathrm{x}$ & $\mathrm{x}$ & & & $\mathrm{x}$ & & $\mathrm{x}$ & \\
\hline 628 & Emberizidae & Buarremon torquatus & $2500-3250$ & $\mathrm{x}$ & & & $\mathrm{x}$ & & & & \\
\hline 629 & Emberizidae & Chlorospingus canigularis & $1000-1600$ & & $\mathrm{x}$ & $\mathrm{x}$ & $\mathrm{x}$ & & & & \\
\hline 630 & Emberizidae & Chlorospingus flavigularis & $800-1600$ & & & $\mathrm{x}$ & $\mathrm{x}$ & & & & \\
\hline 631 & Emberizidae & Chlorospingus ophthalmicus & $1800-2650$ & & $\mathrm{x}$ & & $\mathrm{x}$ & $\mathrm{x}$ & & & \\
\hline
\end{tabular}




\begin{tabular}{|c|c|c|c|c|c|c|c|c|c|c|c|}
\hline 632 & Emberizidae & Chlorospingus parvirostris & $1800-2750$ & $\mathrm{x}$ & $\mathrm{x}$ & & $\mathrm{x}$ & $\mathrm{x}$ & & & \\
\hline 633 & Emberizidae & Lysurus castaneiceps & $900-1800$ & & $\mathrm{x}$ & $\mathrm{x}$ & $\mathrm{x}$ & & & & \\
\hline 634 & Emberizidae & Zonotrichia capensis & $900-4500$ & $\mathrm{x}$ & $\mathrm{x}$ & $\mathrm{x}$ & $\mathrm{x}$ & $\mathrm{x}$ & $\mathrm{x}$ & & $\mathrm{x}$ \\
\hline 635 & Cardinalidae & Chlorothraupis carmioli & $450-1250$ & & & $\mathrm{x}$ & $\mathrm{x}$ & & & & \\
\hline 636 & Cardinalidae & Cyanocompsa cyanoides & $250-1400$ & & & $\mathrm{x}$ & $\mathrm{x}$ & & & & \\
\hline 637 & Cardinalidae & Pheucticus aureoventris & $1800-3200$ & $\mathrm{x}$ & $\mathrm{x}$ & & $\mathrm{x}$ & $\mathrm{x}$ & $\mathrm{x}$ & $\mathrm{x}$ & $\mathrm{x}$ \\
\hline 638 & Cardinalidae & Pheucticus chrysogaster & $650-3500$ & $\mathrm{x}$ & $\mathrm{x}$ & $\mathrm{x}$ & $\mathrm{x}$ & & & & \\
\hline 639 & Cardinalidae & Pheucticus ludovicianus & 2600 & & $\mathrm{x}$ & & $\mathrm{x}$ & & & & \\
\hline 640 & Cardinalidae & Piranga flava lutea & $1800-2700$ & & $\mathrm{x}$ & & $\mathrm{x}$ & $\mathrm{x}$ & & & \\
\hline 641 & Cardinalidae & Piranga leucoptera & $900-1800$ & & $\mathrm{x}$ & $\mathrm{x}$ & $\mathrm{x}$ & & & & \\
\hline 642 & Cardinalidae & Piranga olivacea & $<2000$ & & $\mathrm{x}$ & & $\mathrm{x}$ & $\mathrm{x}$ & & & \\
\hline 643 & Cardinalidae & Piranga rubra & $<3000$ & $\mathrm{x}$ & & & $\mathrm{x}$ & $\mathrm{x}$ & & & \\
\hline 644 & Parulidae & Basileuterus luteoviridis & $2100-3700$ & $\mathrm{x}$ & $\mathrm{x}$ & & $\mathrm{x}$ & $\mathrm{x}$ & $\mathrm{x}$ & & \\
\hline 645 & Parulidae & Basileuterus signatus & $1700-2900$ & $\mathrm{x}$ & $\mathrm{x}$ & & $\mathrm{x}$ & $\mathrm{x}$ & $\mathrm{x}$ & & \\
\hline 646 & Parulidae & Basileuterus tristriatus & $<2200$ & & $\mathrm{x}$ & & $\mathrm{x}$ & $\mathrm{x}$ & & & \\
\hline 647 & Parulidae & Cardellina canadensis & $<2000$ & & $\mathrm{x}$ & & $\mathrm{x}$ & $\mathrm{x}$ & & $\mathrm{x}$ & \\
\hline 648 & Parulidae & Myioborus melanocephalus & $2000-3500$ & $\mathrm{x}$ & $\mathrm{x}$ & & $\mathrm{x}$ & $\mathrm{x}$ & $\mathrm{x}$ & $\mathrm{x}$ & $\mathrm{x}$ \\
\hline 649 & Parulidae & Myioborus miniatus & $1800-2600$ & & $\mathrm{x}$ & & $\mathrm{x}$ & $\mathrm{x}$ & & $\mathrm{x}$ & $\mathrm{x}$ \\
\hline 650 & Parulidae & Myiothlypis chrysogaster & $250-1200$ & & & $\mathrm{x}$ & $\mathrm{x}$ & & & & \\
\hline 651 & Parulidae & Myiothlypis coronata & $1100-2550$ & & $\mathrm{x}$ & $\mathrm{x}$ & $\mathrm{x}$ & $\mathrm{x}$ & & & $\mathrm{x}$ \\
\hline 652 & Parulidae & Myiothlypis luteoviridis & $2500-3700$ & & $\mathrm{x}$ & & & $\mathrm{x}$ & & $\mathrm{x}$ & $\mathrm{x}$ \\
\hline 653 & Parulidae & Myiothlypis signatus & $1800-2800$ & & $\mathrm{x}$ & & & $\mathrm{x}$ & $\mathrm{x}$ & $\mathrm{x}$ & \\
\hline 654 & Parulidae & Phaeothlypis fulvicauda & $250-1500$ & & & $\mathrm{x}$ & $\mathrm{x}$ & & & & \\
\hline 655 & Parulidae & Setophaga cerulea & $<2000$ & & $\mathrm{x}$ & & $\mathrm{x}$ & $\mathrm{x}$ & & & \\
\hline 656 & Parulidae & Setophaga fusca & $<3000$ & $\mathrm{x}$ & $\mathrm{x}$ & & $\mathrm{x}$ & $\mathrm{x}$ & & & \\
\hline 657 & Parulidae & Setophaga pitiayumi & $<2000$ & & $\mathrm{x}$ & & $\mathrm{x}$ & $\mathrm{x}$ & & $\mathrm{x}$ & \\
\hline 658 & Parulidae & Vermivora chrysoptera & 2800 & $\mathrm{x}$ & $\mathrm{X}$ & & $\mathrm{X}$ & & & & \\
\hline
\end{tabular}




\begin{tabular}{|c|c|c|c|c|c|c|c|c|c|c|c|}
\hline 659 & Icteridae & Amblycercus holosericeus & $2100-3300$ & & & & $\mathrm{x}$ & $\mathrm{x}$ & & & \\
\hline 660 & Icteridae & Cacicus chrysonotus & $1800-3450$ & $\mathrm{x}$ & $\mathrm{x}$ & & $\mathrm{x}$ & $\mathrm{x}$ & $\mathrm{x}$ & & $\mathrm{x}$ \\
\hline 661 & Icteridae & Clypicterus oseryi & $1000-3800$ & $\mathrm{x}$ & $\mathrm{x}$ & $\mathrm{x}$ & & & & & $\mathrm{x}$ \\
\hline 662 & Icteridae & Icterus cayanensis & $250-1200$ & & & $\mathrm{x}$ & $\mathrm{x}$ & & & & \\
\hline 663 & Icteridae & Molothrus oryzivorus & $900-1200$ & & & $\mathrm{x}$ & & & & & $\mathrm{x}$ \\
\hline 664 & Icteridae & Psarocolius angustifrons & $1500-2470$ & & $\mathrm{x}$ & & $\mathrm{x}$ & & & $\mathrm{x}$ & $\mathrm{x}$ \\
\hline 665 & Icteridae & Psarocolius atrovirens & $<2600$ & & $\mathrm{x}$ & & $\mathrm{x}$ & $\mathrm{x}$ & & & $\mathrm{x}$ \\
\hline 666 & Fringillidae & Chlorophonia cyanea & $<2100$ & & $\mathrm{x}$ & & $\mathrm{x}$ & $\mathrm{x}$ & & $\mathrm{x}$ & \\
\hline 667 & Fringillidae & Euphonia laniirostris & $<2000$ & & $\mathrm{x}$ & & $\mathrm{x}$ & $\mathrm{x}$ & & & \\
\hline 668 & Fringillidae & Euphonia mesochrysa & $2600-3055$ & $\mathrm{x}$ & $\mathrm{x}$ & & $\mathrm{x}$ & & & $\mathrm{x}$ & \\
\hline 669 & Fringillidae & Euphonia xanthogaster & $<2200$ & & $\mathrm{x}$ & & $\mathrm{x}$ & $\mathrm{x}$ & & $\mathrm{x}$ & $\mathrm{x}$ \\
\hline 670 & Fringillidae & Spinus magellanicus & $900-4200$ & $\mathrm{x}$ & $\mathrm{x}$ & $\mathrm{x}$ & $\mathrm{x}$ & & $\mathrm{x}$ & & \\
\hline 671 & Fringillidae & Spinus olivaceus & $1100-2800$ & $\mathrm{x}$ & $\mathrm{x}$ & & $\mathrm{x}$ & $\mathrm{x}$ & & $\mathrm{x}$ & \\
\hline & & TOTALES & & 251 & 355 & 356 & 584 & 325 & 150 & 131 & 190 \\
\hline
\end{tabular}

